\title{
Os desafios para a efetividade da implementação dos planos de mobilidade urbana: uma revisão sistemática
}

\author{
Challenges for the effectiveness of the implementation of urban mobility plans: \\ a systematic review
}

Laura Machado, Lívia Salomão Piccinini

Universidade Federal do Rio Grande do Sul (UFRGS), Programa de Pós-graduação em Planejamento Urbano e Regional (PROPUR), Porto Alegre, RS, Brasil

\section{Resumo}

Com a criação da lei que instituiu as diretrizes da Política de Mobilidade Urbana brasileira, torna-se necessário investigar os avanços e as barreiras em sua origem: a Comunidade Europeia (CE). Reduzir os impactos dos transportes e alcançar o desenvolvimento urbano sustentável têm motivado a CE a investir em pesquisas e projetos que culminaram com a recomendação para que as cidades adotassem o Sustainable Urban Mobility Plan (SUMP). Embora muitas destas cidades tenham implementado essa proposta, verifica-se, 10 anos depois, que poucos foram os avanços efetivos na redução dos congestionamentos e emissões atmosféricas. Este artigo traz uma revisão sistemática que incluiu 37 estudos sobre o SUMP e apresenta a evolução da política de mobilidade na CE, os guias metodológicos para elaboração, as barreiras encontradas para a implementação e as recomendações para a avaliação dos planos. A revisão mostrou que a metodologia do SUMP foi adotada em outros países fora da CE, como Brasil, México e Índia, sugerindo uma hegemonia no planejamento da mobilidade. Observou-se que uma efetiva implantação do SUMP, depende, além de sua avaliação, do enfrentamento dos desafios colocados frente às decisões políticas sobre as técnicas, da homogeneização socioespacial, da integração entre os níveis de governo, dos projetos setoriais, dos modos de transporte e das medidas propostas.

Palavras-chave: Plano de mobilidade urbana. Políticas públicas. Barreiras. Revisão sistemática.

\section{Abstract}

With the creation of the Law that established the guidelines for the Urban Mobility Policy in Brazil, it became necessary to investigate the progress and the barriers in its origin: the European Community (EC). In order to achieve a sustainable urban development, there has been an increasing interest in reducing the negative impacts of transport in the cities. In this sense, the EC has financed research and projects with the purpose of encouraging the adoption of Sustainable Urban Mobility Plans (SUMP) by all cities. Although many cities have implemented this proposal, after ten years of its creation, minimal changes in congestion and pollutant emissions have been obtained. This paper scrutinizes 37 updated studies that had SUMP as the main subject and that presented the methodologies, barriers and recommendations to evaluate the SUMP. The review

LM é mestre, arquiteta e urbanista, e-mail: laura.lauramachado@gmail.com LSP é doutora, arquiteta e urbanista, e-mail: livia.piccinini@ufrgs.br 
identified the need to overcome relevant barriers in order to achieve an effective implementation of SUMP, which include evaluability, meeting the challenges posed by political decisions on techniques, ensuring socio-spatial homogeneity and, integrating the four levels, i.e. government, sectoral plans, transport modes and measures.

Keywords: Urban mobility plan. Public policies. Barriers. Systematic review.

\section{Introdução}

Transporte é chave para o desenvolvimento das cidades e, paradoxalmente, é responsável por externalidades negativas, que impactam diretamente na economia e na qualidade de vida urbana. Com mais de $80 \%$ da população mundial vivendo em cidades e, na iminência deste percentual atingir 91\% em 2030 (UN, 2014), soluções para a mobilidade desafiam as políticas públicas. Mobilidade tem substituído o termo transporte por transmitir a ideia de deslocamento de pessoas e englobar, além dos sistemas de transporte, os deslocamentos não motorizados. Essa mudança de paradigma está conectada ao desenvolvimento sustentável, discurso hegemônico na esfera mundial desde a publicação do Relatório Burtland, em 1987. Pensar global e agir local tornou-se o lema das Habitat e da Agenda 21, que deram início a um pensamento voltado para a sustentabilidade urbana.

A Comunidade Europeia (CE), na intenção de mitigar os impactos negativos do transporte, tem dado suporte financeiro e técnico a projetos de investigação, desenvolvimento e implementação de medidas de mobilidade concentradas no incentivo ao uso do transporte público e dos meios não motorizados. Investimentos em pesquisa de energias limpas, carros elétricos, uso de tecnologia, gestão de estacionamentos, gerenciamento da mobilidade têm sido realizados desde 1995, com destaque para a iniciativa City Vitalis Sustainability, CiViTAS (Halpern, 2014) e os Planos de Ação (EC, 2009). Essas iniciativas trouxeram avanços importantes na redução de acidentes e avançaram no desenvolvimento de tecnologias para o transporte público, mas não foram suficientemente efetivas para mudar o comportamento das pessoas. Nas cidades europeias, ainda é o automóvel que predomina como meio de deslocamento, prova disso é o aumento de 34\% do número de automóveis em circulação entre 1990 e 2007 (Halpern, 2014). Observe-se que essas iniciativas tampouco alcançaram as metas pretendidas de redução das emissões, dos congestionamentos e dos níveis de mobilidade. A Figura 1 ilustra o aumento das emissões devidas ao transporte na União Europeia.

No Brasil, os números impressionam. A cada ano, 3,5 milhões de veículos novos passam a circular pelas vias urbanas e 43 mil pessoas morrem vítimas de acidentes de trânsito. Trabalhadores perdem, em média, 42,8 minutos/dia no deslocamento casa-trabalho,

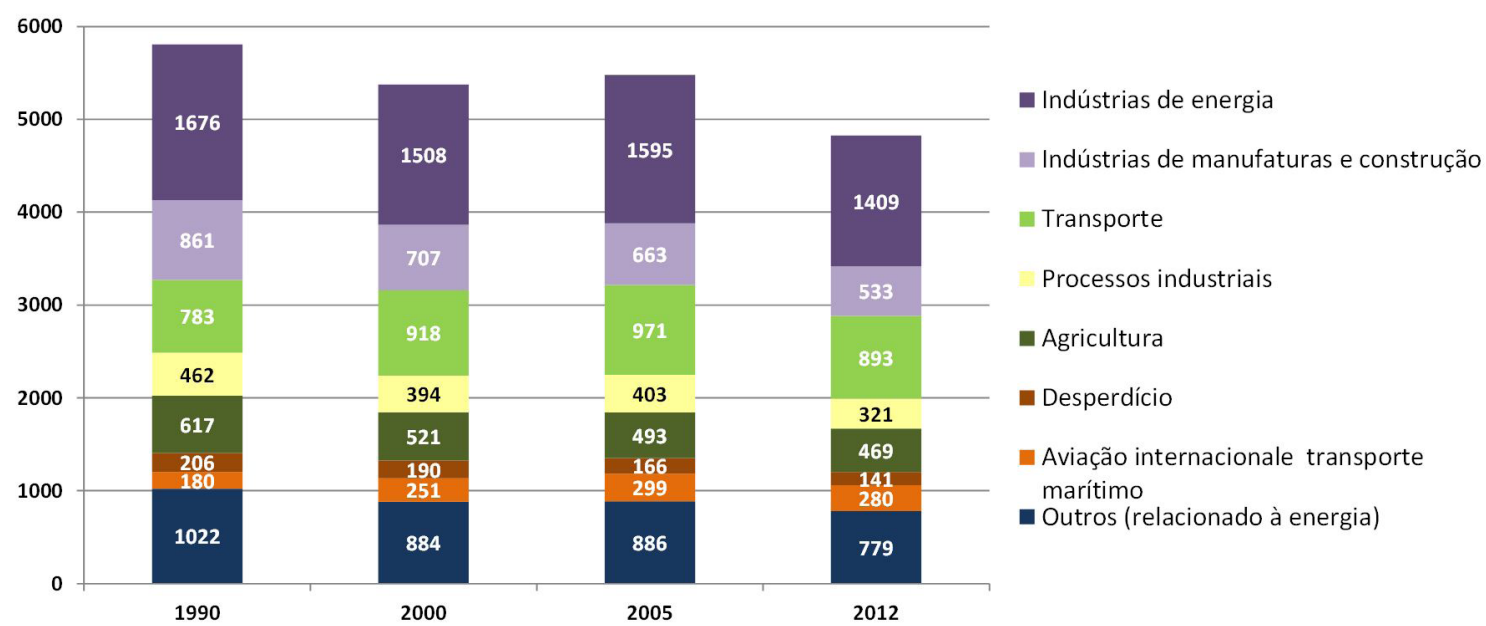

Figura 1 - Emissões de CO² por setor na União Europeia, 1990, 2000, 2005, 2012 (milhões de toneladas por CO² equivalente) Fonte: Adaptado de Eurostat (2016). 
em São Paulo (Brasil, 2015, 2013; Mobilize, 2011). A precariedade da infraestrutura de mobilidade brasileira veio à tona quando o País foi sorteado, em 2007, para sediar a Copa do Mundo de 2014. Em 2008, o Brasil alinhou-se à política internacional de mobilidade através do Projeto Apoio aos Diálogos Setoriais União Europeia-Brasil, coordenado pela Secretaria de Gestão do Ministério do Planejamento. Esse Projeto almejava a transferência de experiências, conhecimento e consolidação dos pactos sociais dos Planos de Mobilidade Urbana Sustentáveis das cidades europeias para as brasileiras (Brasil, 2013). Em 2012, o Governo Federal promulga a Lei 12.587, que estabelece as diretrizes para a Política Nacional de Mobilidade Urbana, centrada no desenvolvimento sustentável das cidades e na priorização dos investimentos federais dos meios não motorizados e dos modos coletivos, incluindo a regulação dos serviços e da política tarifária.

O Plano de Mobilidade Urbana tornou-se o instrumento de efetivação da Política Nacional de Mobilidade (Brasil, 2012). Diante desta determinação, os municípios com mais de vinte mil habitantes estão obrigados a entregar o Plano de Mobilidade Urbana (PMU), como condição para receber recursos federais destinados a projetos de mobilidade urbana. Além de estar integrado ao Plano Diretor de Desenvolvimento Urbano (Brasil 2012), o PMU deve apresentar as ações para materializar os objetivos e metas que pretendem resolver os problemas e as necessidades da população, identificados através de diagnósticos e da participação dos atores sociais (Oliveira, 2012). Também deve contemplar os prazos, especificar as fontes de aporte financeiro, bem como discriminar os indicadores que possibilitarão o monitoramento das ações (Figueiredo \& Figueiredo, 1986; Suárez \& Llaneza, 2013; Wefering et al., 2013; IPEA, 2011).

Apesar do auxílio técnico ofertado pelo Ministério das Cidades (MC), a complexidade da elaboração do PMU exige uma perícia que a maioria dos técnicos municipais das médias e pequenas cidades não possui, o que repercute na baixa proposição realizada pelos municípios. Em abril de 2015, fim do prazo para entrega do PMU (Brasil, 2012), apenas 30\% das cidades haviam entregado seus PMUs. Das cidades que possuem o plano, $69 \%$ são capitais, indicando a dificuldade de atender a essa demanda pelos municípios menos estruturados, os de pequeno e médio porte. Dentre as razões para a baixa aderência, aponta-se a falta de recursos técnicos, financeiros e tecnológicos (NTU, 2015), obrigando os municípios a contratar serviços de consultorias, as quais, em muitos casos, não se mostram adequadas para realizar estudos completos e específicos para cada cidade. Quando houve a obrigatoriedade da elaboração dos Planos Diretores pelo Estatuto das Cidades, muitos planos foram simplesmente replicados de outras cidades e entregues, sem qualquer análise do MC. 0 Ministério tampouco apontou critérios para avaliar os Planos de Mobilidade Urbana (Wefering et al., 2013; Brasil, 2015, 2013; Böhler-Baedeker et al., 2014).

A necessidade de avaliação do PMU fica implícita por ser condição para o recebimento de recursos financeiros governamentais. A avaliação deve apontar coerências e critérios para que os planos sejam factíveis e não fiquem na letra morta. Frente a isso, pergunta-se: Qual é a origem do Sustainable Urban Mobility Plan (SUMP), como foi concebido? Qual é a metodologia proposta para elaborar o plano de mobilidade urbana? Quais são os obstáculos para sua elaboração e implementação? Como são avaliados? A estratégia europeia é válida para as cidades brasileiras? Estas são questões que per se colocam a necessidade de pesquisar a experiência internacional. Considerando-se o contexto e as preocupações apontadas, o objetivo deste artigo é realizar uma revisão sistemática da literatura para compreender a política de mobilidade urbana. Além de artigos publicados em periódicos, foram considerados relatórios de laboratórios de investigação e pesquisa em transportes, práticas, estudos de caso, para embasar a construção de uma metodologia de avaliação dos Planos de Mobilidade Urbana.

\section{Metodologia}

\section{Objetivo e questões que guiam esta pesquisa}

Os objetivos desta revisão são identificar, analisar e resumir o que a literatura apresenta sobre os Planos de Mobilidade Urbana. Com essa determinação, o foco foi dado a artigos e relatórios que investigaram práticas, sistemas de avaliação, políticas, medidas, conceitos, guias e estudos de caso dos Planos de Mobilidade Urbana. Debruçou-se sobre a política de mobilidade europeia por esta ser o discurso dominante e a que influenciou a construção da política de mobilidade brasileira.

Para guiar esta revisão da literatura, foram elaboradas perguntas para possibilitar uma revisão ampla, embora estas não levem, necessariamente, a respostas diretas desta investigação. As questões são:

- Como se deu o processo de evolução da política de transporte que culminou na adoção dos Planos de Mobilidade Urbana? 
- Quais as peculiaridades que caracterizam e distinguem os Planos de Mobilidade Urbana dos planos de transporte tradicionais?

- Qual é a metodologia de elaboração do PMU?

- Quais são as barreiras e dificuldades derivadas das características do PMU, na sua implementação?

- Quais são os critérios para a avaliação da qualidade e da consistência dos Planos de Mobilidade Urbana?

\section{Estratégias de busca}

Esta revisão seguiu os protocolos de revisão sistemática para selecionar, nas bases de dados eletrônicas, artigos e relatórios relevantes. Primeiramente, foram identificadas palavras-chave que conseguissem captar a problemática de pesquisa relativa aos Planos de Mobilidade Urbana. Uma ampla escolha de base de dados foi selecionada a fim de cobrir artigos de periódicos e relatórios de pesquisa publicados. Foram consultadas bases de dados de periódicos (Web of Science, Ebsco, Scopus, Springer), bases de dados de laboratórios de pesquisa em transporte (Transport Research Board - TRB, National Transport Library, World Transport Research, Transport Research Portal, Institute for Transport Studies Leeds, European Commission) e os sites Eltis - The urban mobility observatory (Eltis, 2016) e Polis European cities and regions networking from innovative transportsolutions (POLIS, 2016). Foram identificadas palavras-chave associadas diretamente com as questões de pesquisa: urban mobility; mobility polic*; mobility plan; mobility criteria, que foram combinadas com termos como review; method*; criteria; evaluat*. A captura de artigos e relatórios restringiu-se àqueles escritos na língua inglesa, nas seguintes áreas de estudo: transporte, planejamento urbano, ciências sociais e políticas públicas. Não foi estabelecida data inicial para o início da busca.

De uma lista inicial de 3.200 títulos, editada pela leitura dos abstracts e pertinência à temática "Avaliação dos Planos de Mobilidade Urbana", restaram 195 estudos que foram incorporados ao software Mendeley, para facilitar a exclusão de duplicidades e realizar uma leitura transversal. Destes, 151 foram excluídos por não incluir pesquisa que envolvesse Plano de Mobilidade ou de Transporte. Dos 44 documentos, sete documentos foram excluídos, quais sejam: dois, por descreverem a mesma pesquisa (May et al., 2008; May, 2009; López-Lambas et al., 2010, 2012); os estudos de May et al. (2012) e Vieira et al. (2007) foram retirados por tratarem de metodologias para selecionar medidas (estratégias); os estudos de Dziekan et al. (2013) e de Browne \& Ryan (2011), por apresentarem técnicas de avaliação de medidas, e o relatório apresentado por Lopez-Ruiz et al. (2013), por se restringir aos resultados obtidos em diferentes medidas adotadas. Com a aplicação deste segundo filtro, 37 documentos (16 papers e 21 relatórios) foram considerados relevantes (Figura 2).

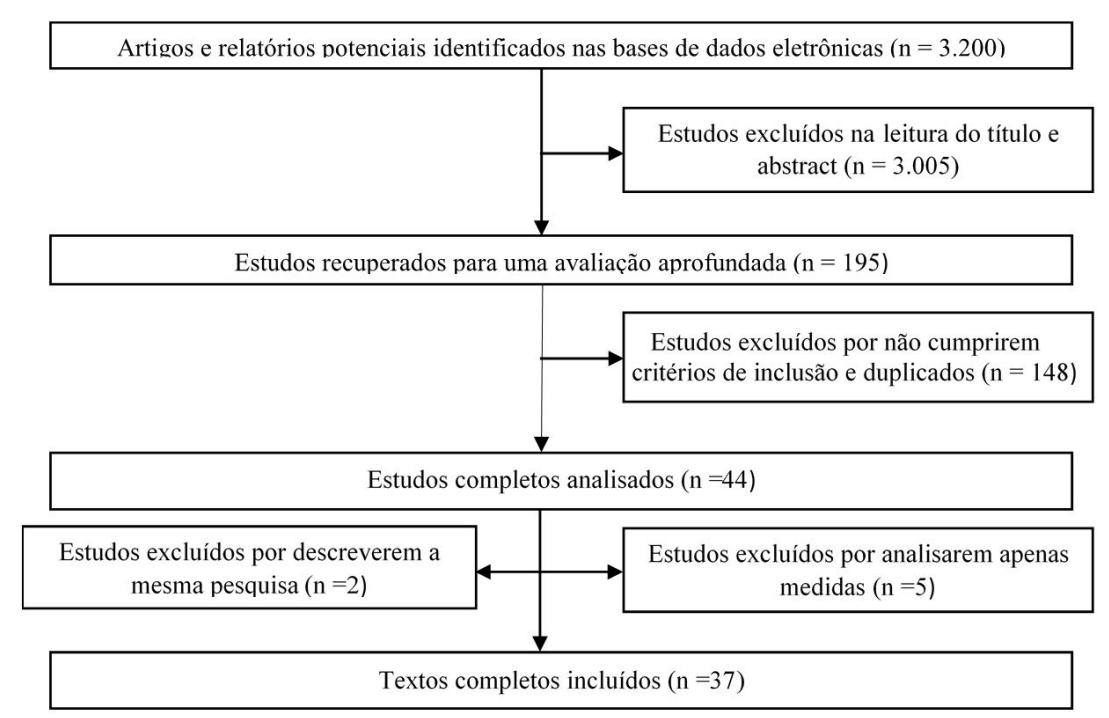

Figura 2 - Fluxograma da revisão sistemática Fonte: Elaboraç̃õo da autora. 


\section{Resultados}

Relatórios e estudos sobre Planos de Mobilidade (Tabela 1) foram encontrados a partir de publicações do ano 2000 e originam-se, em sua maioria, nos países Bélgica (38\%), Inglaterra (22\%) e Alemanha (16\%).
Observa-se um crescimento nesta temática a partir do ano 2010.

Os estudos foram analisados por temática específica das questões que se quer responder (Tabela 2) e que refletem o quadro de pesquisa atual: (i) a política de transporte e mobilidade da Comunidade Europeia;

Tabela 1 - Identificação de número de estudos por período de publicação, autor, ano e país de origem

\begin{tabular}{|c|c|c|c|c|}
\hline Autores (Ano) & País & $2000-05$ & 2006-10 & 2011-15 \\
\hline Baeten (2000) & Reino Unido & & & \\
\hline European Comission (EC, 2001a) & Bélgica & & & \\
\hline European Commission (EC, 2001b) & Bélgica & & & \\
\hline May et al. (2003) & Reino Unido & & & \\
\hline May (2003) & Reino Unido & & & \\
\hline Minken et al. (2003) & Reino Unido & 11 & & \\
\hline Department for Transport (DFT, 2004) & Reino Unido & & & \\
\hline Diamantini \& Geneletti (2004) & Itólia & & & \\
\hline European Commission (EC, 2004) & Bélgica & & & \\
\hline Wolfram (2004) & Alemanha & & & \\
\hline Hull (2005) & Reino Unido & & & \\
\hline European Commission (EC, 2007a) & Bélgica & & & \\
\hline European Commission (EC, 2007b) & Bélgica & & & \\
\hline European Commission (EC, 2007c) & Bélgica & & & \\
\hline European Commission (EC, 2009) & Bélgica & & 7 & \\
\hline May (2009) & Reino Unido & & & \\
\hline López-Lambas et al. (2010) & Espanha & & & \\
\hline Trasporti e Territorio (TRT, 2010) & Bélgica & & & \\
\hline European Commission (EC, 2011) & Bélgica & & & \\
\hline Gil et al. (2011) & Portugal & & & \\
\hline Hrelia (2011) & Suécia & & & \\
\hline European Commission (EC, 2012) & Bélgica & & & \\
\hline Racero et al. (2012) & Espanha & & & \\
\hline European Commission (EC, 2013a) & Bélgica & & & \\
\hline European Commission (EC, 2013b) & Bélgica & & & \\
\hline Halpern (2014) & França & & & \\
\hline Kastelic et al. (2013) & Áustria & & & \\
\hline López-Lambas et al. (2012) & Espanha & & & 19 \\
\hline Poppeliers \& Ricci (2013) & Holanda & & & \\
\hline Tormans et al. (2013) & Bélgica & & & \\
\hline Wefering et al. (2013) & Alemanha & & & \\
\hline Böhler-Baedeker et al. (2014) & Alemanha & & & \\
\hline Burggraf et al. (2014) & Alemanha & & & \\
\hline Keblowski et al. (2014) & Bélgica & & & \\
\hline Lindenau et al. (2014) & Alemanha & & & \\
\hline Rudolph, F. et al. (2014) & Alemanha & & & \\
\hline May (2015) & Reino Unido & & & \\
\hline
\end{tabular}

Fonte: Elaboração própria. 
Tabela 2 - Número de estudos encontrados por temas e por autor

\begin{tabular}{|c|c|c|c|}
\hline Tema & Autores & $\mathrm{N}^{\circ}$ estudos & $\%$ \\
\hline Política transporte CE & EC (2001b, 2004, 2007b, 2009, 2011, 2012, 2013b); Halpern (2014); Poppeliers \& Ricci (2013) & 10 & $37 \%$ \\
\hline Metodologias de elaboração do plano & $\begin{array}{l}\text { May (2003); Wolfram (2004); DFT (2004); EC (2007c); Wefering et al. (2013); Böhler-Baede- } \\
\text { ker et al. (2014); Lindenau et al. (2014); Racero et al. (2012) }\end{array}$ & 8 & $22 \%$ \\
\hline Barreiras à implementação do plano & $\begin{array}{l}\text { Baeten (2000); May (2009); May et al. (2001, 2003, 2006); Diamantini \& Geneletti (2004); Hull } \\
\text { (2005); LópezzLambas et al. (2010); Hrelia (2011); Tormans et al. (2013); Keblowski et al. (2014) }\end{array}$ & 12 & $33 \%$ \\
\hline Avaliação do plano & $\begin{array}{l}\text { Burggraf et al. (2014), DFT (2004); Diamantini \& Geneletti (2004); Gil et al. (2011); } \\
\text { Hrelia (2011); Kastelic et al. (2013), López-Lambas et al. (2012); Rudolph et al. (2014); } \\
\text { Tormans et al. (2013); Wefering et al. (2013) }\end{array}$ & 10 & $27 \%$ \\
\hline
\end{tabular}

Fonte: Elaboracãa da autora.

(ii) as barreiras para a implementação do Plano de Mobilidade; (iii) as metodologias para elaboração e implementação do Plano; (iv) a avaliação dos Planos de Mobilidade.

\section{Evolução da política de transporte europeia}

Antes mesmo da assinatura do Tratado de Maastricht, em 1992, ou seja, antes de existir a União Europeia, a França já havia criado o Plan de Déplacements Urbains (PDU), em 1982. Logo após, outros países estabeleceram seus planos de transporte: a Alemanha com o Verkehrsentwicklungsplan (VEP - 1994), a Bélgica com o Mobility Plan (MP - 1996), a Inglaterra com o Local Transport Plan (LTP - 2000), a Itália com o Piano Urbano della Mobilitá (PUM - 2000) e a Hungria (Transport Development Plan - 2001) (López-Lambas \& Leániz, 2010; Wefering et al., 2013). Estes planos, em um primeiro momento, possuíam um caráter conservador, voltados exclusivamente às questões de trânsito e transporte, da infraestrutura e da primazia do automóvel, que resultaram na separação física entre as pessoas e o tráfego (Keblowski et al., 2014; López-Lambas \& Leániz, 2010; Tormans et al., 2013).

A partir dos anos 1990, pressões internacionais comprometeram os países a cumprirem acordos e protocolos pelo clima e pela sustentabilidade. A mobilidade torna-se um elemento-chave para a redução das emissões e demais externalidades advindas do transporte. Muda-se o paradigma da mobilidade: o automóvel é combatido e os meios não motorizados e o transporte público tornam-se protagonistas, uma solução para os problemas urbanos. A Tabela 3 descreve as características fundamentais dos paradigmas do planejamento do transporte, acrescentando um terceiro: o crítico.

O terceiro paradigma está colocado como um contraponto aos paradigmas anteriores justamente para apontar a necessidade de mudanças estruturais no transporte urbano. A perspectiva crítica levanta questões sobre quais são os interesses que estão sendo atendidos pelas políticas e práticas do transporte. No planejamento tradicional, não estão colocadas as diferenças entre os altos padrões da mobilidade dos ricos e a baixa mobilidade dos pobres, tampouco a desigualdade de cada estrato social na contribuição para os impactos ambientais ou até mesmo as diferenças socioespaciais e a falta de vontade política em reduzir a mobilidade (Keblowski et al., 2014).

Na literatura, o paradigma da sustentabilidade é a política oficial para as cidades da CE (EC, 2001a, 2001b, 2007b, 2009). Os relatórios e comunicações da CE permitem compreender a evolução de sua política: de um planejamento regional, fundamental para o desenvolvimento econômico, voltada ao atendimento do mercado, para a satisfação das demandas e para o incremento da conectividade e da competição (Baeten, 2000; EC, 2001a, 2001b), a CE entende que o desafio de resolver os conflitos da mobilidade se dá nas cidades (EC, 2007b, 2013b; Halpern, 2014).

A mobilidade urbana integrada, limpa e segura torna-se, portanto, uma das prioridades na política europeia, alicerçada por altos investimentos no setor. Desde 1995, 10 bilhões de euros financiaram programas de fomento de projetos para a mobilidade sustentável (Halpern, 2014); deste total, 300 milhões de euros foram investidos em pesquisas em transporte urbano (Poppeliers \& Ricci, 2013). Dentre outros programas, 
Tabela 3 - Paradigmas do planejamento do transporte urbano

\begin{tabular}{|c|c|}
\hline Paradigma & Características \\
\hline \multirow{6}{*}{ Ortodoxo- Tradicional } & Foco no trânsito, fluxo, capacidade e velocidade \\
\hline & Foco no modo rodoviário e na infraestrutura \\
\hline & No nível sistêmico: perícia, técnica e racionalidade \\
\hline & Transporte como motor da economia: congestionamento que leva a tempo de viagem improdutivo \\
\hline & Uso de modelagem computacional e cenários; análise custo-benefício como principal ferramenta de avaliação \\
\hline & Zoneamento, uso do solo monofuncional, planejamento orientado ao uso do automóvel \\
\hline \multirow{9}{*}{ Sustentável } & Foco nas pessoas \\
\hline & Acessibilidade e qualidade de vida \\
\hline & Equilibrio entre os meios de transporte e mudança para os mais sustentáveis \\
\hline & Quebra da perspectiva puramente econômica dos transportes; inclusão de aspectos sociais e ambientais \\
\hline & $\begin{array}{l}\text { Transporte como componente de uma "boa cidade": economicamente produtiva; coesão e diversidade social; maiores densidades e uso misto do solo; } \\
\text { amiga do ambiente; saudável e participativa }\end{array}$ \\
\hline & Direcionado a transporte público e meios nõo motorizados \\
\hline & Forte integração entre uso do solo e transporte \\
\hline & Maior atenção para o comportamento individual e estilo de vida \\
\hline & Formas mais participativas de criar políticas e práticas de transporte \\
\hline \multirow{9}{*}{ Crítico } & Crítica aos paradigmas tradicional e sustentável por sua visão técnica, quantitativa, descritiva e despolitizada \\
\hline & Crítica às soluções tecnológicas e comportamentais para problemas sociais e políticos estruturais do transporte \\
\hline & Ampliação da visão política e social \\
\hline & Crítica à falta de enfrentamento das razões sistêmicas por trás da insustentabilidade \\
\hline & Crítica à eufemização e individualização das causas estruturais dos problemas da mobilidade \\
\hline & Foco nas relações sociais, econômicas e políticas, e regulamentação das estruturas de transporte \\
\hline & Reconhecimento das questões de gênero, raça, etnia, classe, deficientes e de idade \\
\hline & Reconhecimento da mobilidade como uma norma e forma discriminatória do capital produzir iniquidades socioespaciais \\
\hline & Crítica das práticas empresariais no transporte \\
\hline
\end{tabular}

Fonte: Böhler-Baedeker et al. (2014); Keblowski et al. (2014).

a iniciativa City Vitalis Sustainability (CiViTAS) é o seu maior representante (EC, 2013b; Halpern, 2014). Em suas quatro edições, foram desenvolvidos projetos que buscam soluções para a mobilidade (Tabela 4), principalmente no que tange a veículos inteligentes, novas tecnologias, inovações e gerenciamento da mobilidade.

Além das iniciativas CiViTAS, a CE financiou programas específicos envolvendo a coordenação do planejamento de transporte com o uso do solo: Land Use and Transport Research Cluster (LUTR), TRANSport, Land Use and Sustainability (TRANSPLUS), Procedures for Recommending Sustainable Planning of European City Transport Systems (PROSPECTS), Integration of
Transport and Land Using Planning (TRANSLAND), Sprawling Cities and Transport: From Evaluation to Recommendations (SCATTER), Planning and Research Of Policies Land use and transport for Increasing urban Sustainability (PROPOLIS), COST332-Transport and Land use Policies (May, 2003; EC, 2001a, 2004, 2007a; TRT, 2010). Esses programas tinham como escopo medidas de restrição do uso do automóvel, recuperação de áreas centrais através de conexões com o transporte público e meios não motorizados, compacidade e diversificação urbana (EC, 2007a, 2007b).

Porém, a constatação de que medidas isoladas, pontuais e de curto prazo não eram suficientes para alterar os padrões de mobilidade ou mesmo 
minimizar as externalidades negativas, indicou-se a necessidade de que as cidades elaborassem os Planos de Transporte Urbano Sustentável (EC, 2004, 2007c), que logo foram substituídos pelos Planos de Mobilidade Urbana Sustentável (PMUS ou SUMP na sua sigla em inglês) (EC, 2011, 2012, 2013a; Poppeliers \& Ricci, 2013). A Figura 3 apresenta a linha do tempo - das principais resoluções clarificando a trajetória da política europeia, que se iniciou com medidas de mobilidade avulsas que culminaram na recomendação da adoção do PMUS, objeto deste estudo.

Planos de mobilidade urbana: conceito e elaboração

A definição do conteúdo necessário ao desenvolvimento do PMUS foi construída a partir de estudos e projetos conduzidos por especialistas (EC, 2013a), como

Tabela 4 - Iniciativas CiViTAS, projetos e cidades participantes

\begin{tabular}{cccc}
\hline $\begin{array}{c}\text { CiVitaS I } \\
\mathbf{( 2 0 0 2 - 2 0 0 6 )}\end{array}$ & $\begin{array}{c}\text { CiVitAS II } \\
\mathbf{( 2 0 0 5 - 2 0 0 9 )}\end{array}$ & $\begin{array}{c}\text { CiViTAS Plus } \\
\mathbf{( 2 0 0 8 - 2 0 1 2 )}\end{array}$ & $\begin{array}{c}\text { CiVitAS Plus II } \\
\mathbf{( 2 0 1 2 - 2 0 1 6 )}\end{array}$ \\
\hline 19 cidades & 17 cidades & 25 cidades & 8 cidades \\
4 Projetos & 4 Projetos & 5 Projetos & 2 Projetos \\
Miracles, Tellus, Trendsetter e Vivaldi & Caravel, Mobilis, Smile e Success & Archimedes, Elan, Mimosa, Modern e Renaissance & Din@amo e 2Move2 \\
Custo total dos projetos & & & $€ 300$ milhões \\
\hline
\end{tabular}

Fonte: Adaptado de Roojen \& Nesterova (2013).

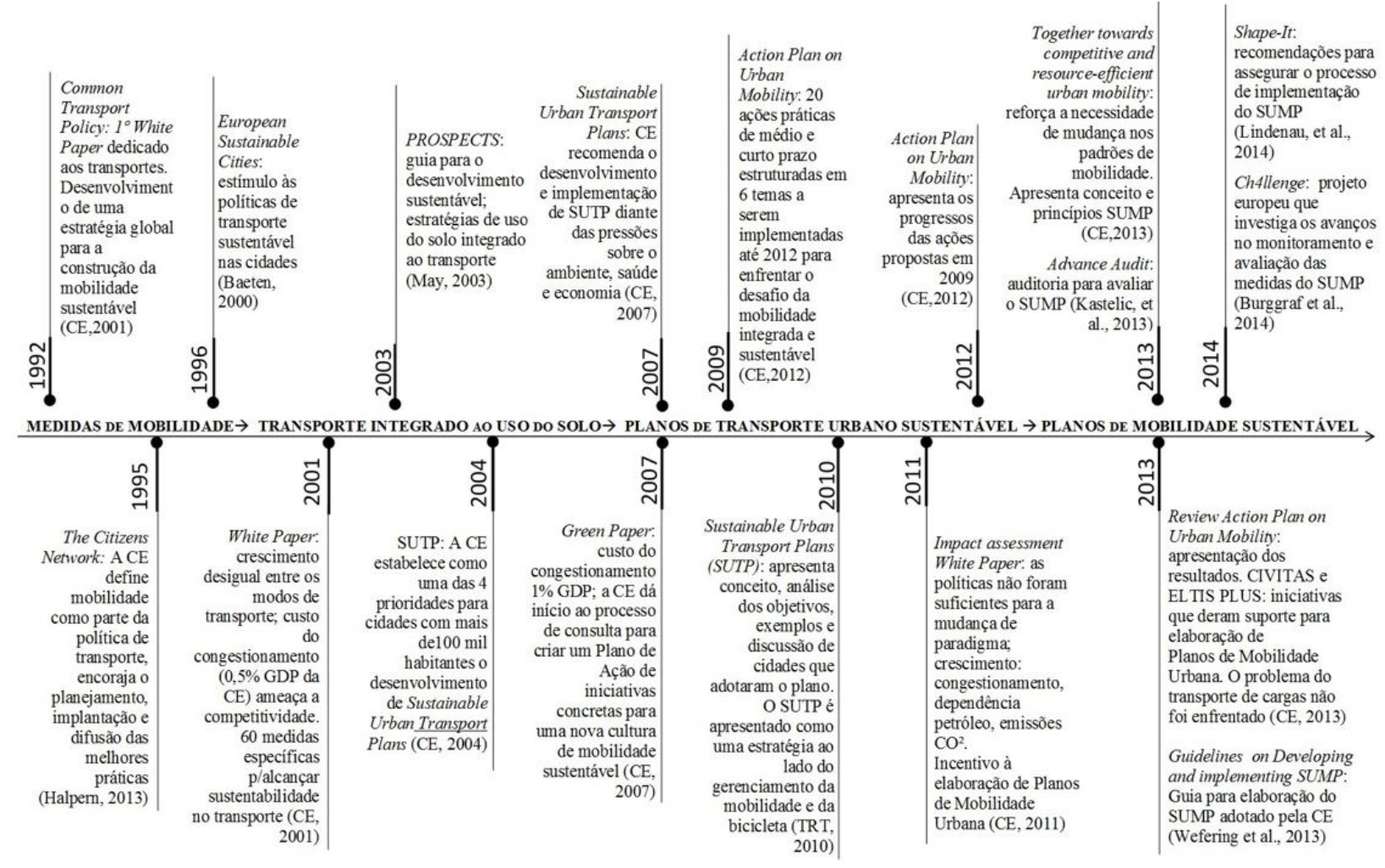

Figura 3 - Linha do tempo da formação da política de mobilidade europeia Fonte: Elaboraç̃o da autora. 
exposto anteriormente. Em sua concepção, o PMUS é um conjunto de medidas inter-relacionadas projetadas para satisfazer as necessidades de deslocamento de pessoas e mercadorias hoje e no futuro (Kastelic et al., 2013). Para ser caracterizado como sustentável, o plano deve ter por objetivos melhorar a acessibilidade, a integração, a eficiência e a qualidade dos meios de transporte, e reduzir as emissões e os acidentes.

0 processo de construção do plano se inicia com a composição de uma equipe transdisciplinar, que irá realizar o diagnóstico da situação atual, considerando e integrando os demais planos existentes - como o de desenvolvimento urbano - e o levantamento das necessidades identificadas pelos diversos atores que compõem a sociedade organizada. 0 cidadão deve ser chamado a participar e expor suas necessidades de mobilidade e acessibilidade.

A consecução do plano depende da implementação de ações que devem estar alicerçadas por cenários, avaliações de custos e definições das fontes de financiamento, e monitoradas por indicadores, e depende também da definição do responsável pela ação, ou seja, pela gestão do plano. As propostas do plano devem equilibrar e integrar os diferentes meios de transporte e obter a cooperação dos diferentes níveis de governo. As ações devem ser monitoradas e revisadas caso necessário e, os resultados, devem ser comunicados à sociedade. A competência da implantação, bem como o estabelecimento de mecanismos para garantir a qualidade e a validade do PMUS dentro da esfera da sustentabilidade, fica a cargo da autoridade local (EC, 2013b; Kastelic et al., 2013). A Figura 4 ilustra o ciclo de elaboração do Plano de Mobilidade Urbana Sustentável (PMUS) elaborado pela União Europeia.

De uma forma simplificada, a elaboração do PMUS pressupõe um plano estratégico pensado para atingir

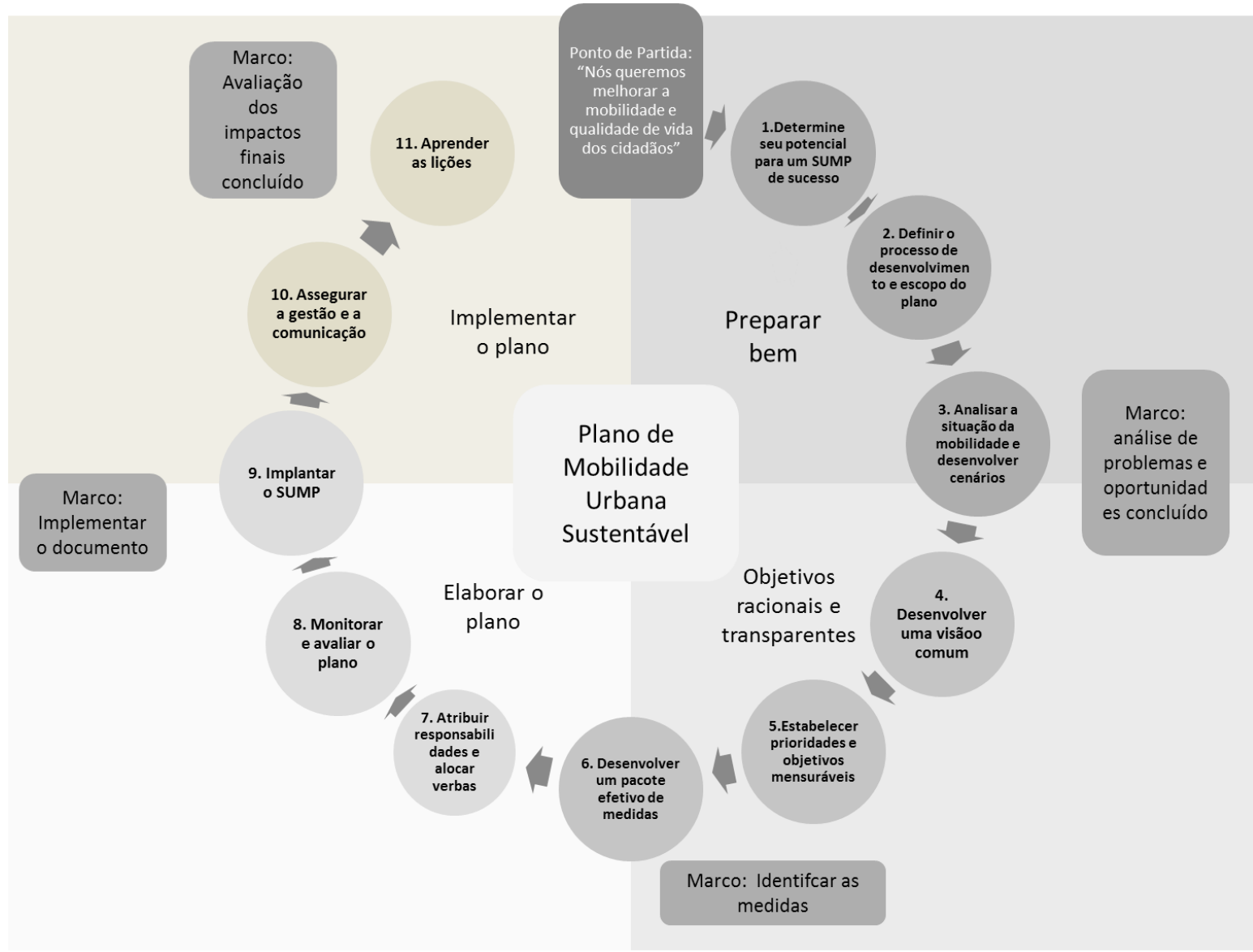

Figura 4 - Ciclo de elaboraç̃ão do SUMP Fonte: Adaptado de Wefering et al. (2013). 
resultados consistentes, com uma missão e objetivos definidos em conjunto com os atores (participação), para alcançar um modelo de cidade futura (visão), em um determinado período de tempo (prazo) monitorado por indicadores. Essa metodologia, bem como as medidas sugeridas, são comuns nos guias de elaboração encontrados na literatura (Tabelas 5 e 6) (Minken etal., 2003; May, 2003; DFT, 2004; Wolfram etal., 2005; EC, 2007a, 2007c; Wefering et al., 2013), e é seguida por diversos países, mesmo fora da Europa (Kastelic et al., 2013; Böhler-Baedeker et al., 2014; Lindenau et al., 2014).

Tabela 5 - Características, etapas para elaboração do SUMP encontradas nos manuais e países que 0 adotaram

\begin{tabular}{|c|c|c|c|c|c|c|c|c|c|c|c|c|c|}
\hline & \multicolumn{6}{|c|}{ Manuais de elaboração } & \multicolumn{7}{|c|}{ Planos de Mobilidade e transporte adotados } \\
\hline & 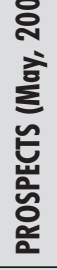 & 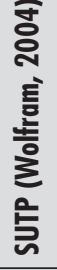 & 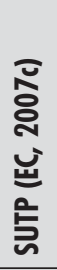 & 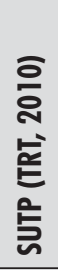 & 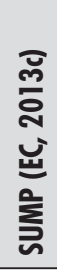 & 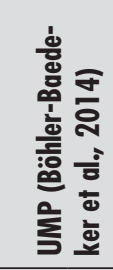 & $\begin{array}{l}\text { 흫 } \\
\text { 흫 } \\
\text { 흘 } \\
\text { 틀 }\end{array}$ & $\begin{array}{l}\text { 를 } \\
\text { 폰 } \\
\text { 를 }\end{array}$ & 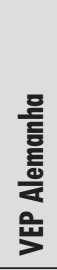 & 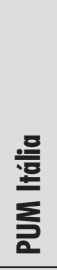 & $\frac{\frac{\text { 흘 }}{\text { 드 }}}{\text { 른 }}$ & 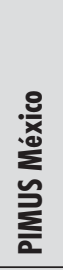 & 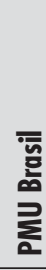 \\
\hline \multicolumn{14}{|l|}{ Elementos-chave - características } \\
\hline Participaç̃̃o & $\checkmark$ & $\checkmark$ & $\checkmark$ & $\checkmark$ & $\checkmark$ & $\checkmark$ & $\checkmark$ & $\checkmark$ & $\checkmark$ & $\checkmark$ & $\checkmark$ & & $\checkmark$ \\
\hline Definição de horizonte - longo prazo & $\checkmark$ & $\checkmark$ & & $\checkmark$ & $\checkmark$ & & $\checkmark$ & $\checkmark$ & & $\checkmark$ & & $\checkmark$ & $\checkmark$ \\
\hline Ações baseadas em melhores práticas & $\checkmark$ & $\checkmark$ & $\checkmark$ & $\checkmark$ & $\checkmark$ & $\checkmark$ & & & & & & & \\
\hline Integração com demais políiticas (usos do solo) & $\checkmark$ & $\checkmark$ & $\checkmark$ & $\checkmark$ & $\checkmark$ & $\checkmark$ & $\checkmark$ & & $\checkmark$ & & $\checkmark$ & $\checkmark$ & $\checkmark$ \\
\hline Integração com outros níveis de governo & $\checkmark$ & $\checkmark$ & $\checkmark$ & $\checkmark$ & $\checkmark$ & $\checkmark$ & $\checkmark$ & & $\checkmark$ & & & $\checkmark$ & $\checkmark$ \\
\hline Integração modos e meios & $\checkmark$ & & $\checkmark$ & $\checkmark$ & $\checkmark$ & $\checkmark$ & $\checkmark$ & & $\checkmark$ & & & & $\checkmark$ \\
\hline Objetivos alinhados com sustentabilidade & $\checkmark$ & $\checkmark$ & $\checkmark$ & $\checkmark$ & $\checkmark$ & & $\checkmark$ & & $\checkmark$ & & & & \\
\hline Identificação das necessidades atuais e futuras & $\checkmark$ & $\checkmark$ & & $\checkmark$ & $\checkmark$ & $\checkmark$ & $\checkmark$ & & & & & & \\
\hline Uso de indicadores & $\checkmark$ & $\checkmark$ & & & $\checkmark$ & $\checkmark$ & $\checkmark$ & $\checkmark$ & & $\checkmark$ & $\checkmark$ & & $\checkmark$ \\
\hline Suporte financeiro & & $\checkmark$ & & & $\checkmark$ & & $\checkmark$ & & & & $\checkmark$ & $\checkmark$ & $\checkmark$ \\
\hline Monitoramento, revisão e comunicação & $\checkmark$ & $\checkmark$ & $\checkmark$ & $\checkmark$ & $\checkmark$ & $\checkmark$ & $\checkmark$ & & & & $\checkmark$ & & $\checkmark$ \\
\hline \multicolumn{14}{|l|}{ Passos para elaboração } \\
\hline Preparação & $\checkmark$ & $\checkmark$ & $\checkmark$ & & $\checkmark$ & & $\checkmark$ & & & & $\checkmark$ & & $\checkmark$ \\
\hline Diagnóstico da situação atual & $\checkmark$ & $\checkmark$ & $\checkmark$ & & $\checkmark$ & $\checkmark$ & $\checkmark$ & $\checkmark$ & $\checkmark$ & & $\checkmark$ & & $\checkmark$ \\
\hline Definição da visão, objetivos e metas mensuráveis & $\checkmark$ & $\checkmark$ & $\checkmark$ & & $\checkmark$ & $\checkmark$ & $\checkmark$ & $\checkmark$ & & $\checkmark$ & $\checkmark$ & $\checkmark$ & $\checkmark$ \\
\hline Elaboração de cenários & $\checkmark$ & $\checkmark$ & & & $\checkmark$ & $\checkmark$ & & $\checkmark$ & & & $\checkmark$ & & $\checkmark$ \\
\hline Definição de pacote de medidas/ações & $\checkmark$ & $\checkmark$ & $\checkmark$ & & $\checkmark$ & & & $\checkmark$ & $\checkmark$ & $\checkmark$ & $\checkmark$ & $\checkmark$ & $\checkmark$ \\
\hline Previsão de impactos adversos & $\checkmark$ & & & & $\checkmark$ & & & & $\checkmark$ & & & & \\
\hline Identificar custos das medidas/orçamentos & $\checkmark$ & $\checkmark$ & & & $\checkmark$ & $\checkmark$ & $\checkmark$ & $\checkmark$ & $\checkmark$ & & $\checkmark$ & $\checkmark$ & $\checkmark$ \\
\hline Identificar responsabilidades/gestão & & $\checkmark$ & & & $\checkmark$ & & & & & & & $\checkmark$ & \\
\hline Otimização (encontrar melhor estratégia) & $\checkmark$ & & & & $\checkmark$ & $\checkmark$ & $\checkmark$ & $\checkmark$ & & & $\checkmark$ & & \\
\hline Implementaç̃̃o & $\checkmark$ & $\checkmark$ & $\checkmark$ & & $\checkmark$ & $\checkmark$ & $\checkmark$ & & $\checkmark$ & & $\checkmark$ & & $\checkmark$ \\
\hline Monitoramento e comunicação & $\checkmark$ & $\checkmark$ & $\checkmark$ & & $\checkmark$ & $\checkmark$ & $\checkmark$ & & $\checkmark$ & & $\checkmark$ & & $\checkmark$ \\
\hline Revisão & $\checkmark$ & & $\checkmark$ & & $\checkmark$ & & $\checkmark$ & & & & $\checkmark$ & & $\checkmark$ \\
\hline
\end{tabular}

Fonte: Elaboração da autora. 
Tabela 6 - Medidas sugeridas para 0 SUMP encontradas nos manuais e nos países que 0 adotaram

\begin{tabular}{|c|c|c|c|c|c|c|c|c|c|c|c|}
\hline & 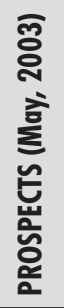 & 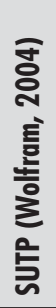 & 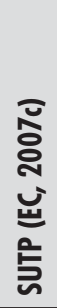 & 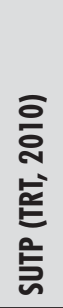 & 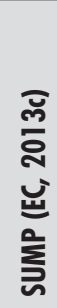 & 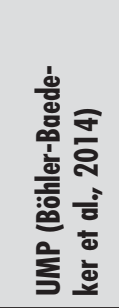 & 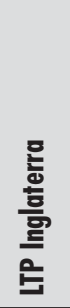 & 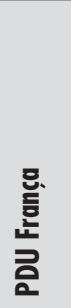 & 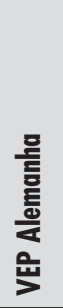 & $\frac{\text { 를 }}{\text { 吾 }}$ & 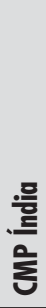 \\
\hline \multicolumn{12}{|l|}{ Medidas e objetivos sugeridos } \\
\hline Coordenacçõo entre uso do solo e transporte & $\checkmark$ & $\checkmark$ & $\checkmark$ & & $\checkmark$ & & $\checkmark$ & & & & $\checkmark$ \\
\hline Promoção e incentivo do transporte coletivo & $\checkmark$ & $\checkmark$ & $\checkmark$ & & $\checkmark$ & $\checkmark$ & $\checkmark$ & $\checkmark$ & & $\checkmark$ & $\checkmark$ \\
\hline Incentivar meios ñ̃o motorizados (walk and bike) & $\checkmark$ & $\checkmark$ & $\checkmark$ & & $\checkmark$ & $\checkmark$ & $\checkmark$ & $\checkmark$ & & $\checkmark$ & $\checkmark$ \\
\hline Gerenciamento do transporte de cargas & $\checkmark$ & $\checkmark$ & $\checkmark$ & & $\checkmark$ & & $\checkmark$ & $\checkmark$ & & & $\checkmark$ \\
\hline Gerenciamento dos estacionamentos & & $\checkmark$ & $\checkmark$ & & $\checkmark$ & $\checkmark$ & & $\checkmark$ & & $\checkmark$ & $\checkmark$ \\
\hline Pedágios urbanos & & & $\checkmark$ & & $\checkmark$ & & $\checkmark$ & & & & \\
\hline Traffic calming & & & $\checkmark$ & & $\checkmark$ & & & & & & \\
\hline Restriç̣̃o para veículos mais poluentes & & & $\checkmark$ & & $\checkmark$ & & $\checkmark$ & & & & \\
\hline Fomentar veículos limpos, silenciosos, baixas emissões & & & $\checkmark$ & & $\checkmark$ & & $\checkmark$ & $\checkmark$ & & & $\checkmark$ \\
\hline Medidas soft (car-sharing, school travel plans, MM) & & $\checkmark$ & $\checkmark$ & & $\checkmark$ & & $\checkmark$ & $\checkmark$ & & $\checkmark$ & \\
\hline Medidas de uso do solo & $\checkmark$ & $\checkmark$ & & & $\checkmark$ & & & & & & \\
\hline Redução do número de viagens & $\checkmark$ & & & & $\checkmark$ & & $\checkmark$ & & & & \\
\hline Redução do uso do automóvel & $\checkmark$ & $\checkmark$ & & & $\checkmark$ & & $\checkmark$ & $\checkmark$ & & $\checkmark$ & $\checkmark$ \\
\hline Acessibilidade universal & $\checkmark$ & $\checkmark$ & & & $\checkmark$ & & & $\checkmark$ & & & \\
\hline Redução de acidentes & & $\checkmark$ & & & $\checkmark$ & & $\checkmark$ & $\checkmark$ & & $\checkmark$ & \\
\hline Redução das emissões & & $\checkmark$ & & & $\checkmark$ & & $\checkmark$ & $\checkmark$ & & $\checkmark$ & \\
\hline Medidas para promover inclusão social & & $\checkmark$ & & & $\checkmark$ & & $\checkmark$ & & & & $\checkmark$ \\
\hline Planejamento e gerenciamento da rede viária & $\checkmark$ & $\checkmark$ & & & $\checkmark$ & $\checkmark$ & $\checkmark$ & & & & $\checkmark$ \\
\hline
\end{tabular}

Fonte: Elaboraç̃o da autora.

Racero et al. (2012) propõem o uso de softwares gratuitos de informação geográfica, para auxiliar na elaboração e padronização dos PMUs, uma vez que i) oferecem suporte e visualização dos dados e indicadores; ii) permitem realizar diagnósticos, análises e monitoramento, e iii) ainda possibilitam a participação dos cidadãos. Como o estudo, conduzido para a área metropolitana de Sevilla, está em andamento, não foram apresentados resultados.

\section{Barreiras à implementação do PMUS}

A implementação das estratégias do Plano não é uma tarefa simples, pois é mais fácil falar do que fazer (May et al., 2008). 0 sistema de transporte é o resultado de uma produção em cadeia que não é controlado por uma organização, mas é o resultado de uma interação, que envolve diferentes organismos, instituições e pessoas, dentro de um ambiente vivo, que é o espaço urbano (Tormans et al., 2013).

Para haver transformações efetivas na mobilidade urbana, é necessário um esforço político para promover as diversas formas de integração, superar as barreiras externas e melhorar os processos organizacionais e técnicos. Nesta revisão, 11 estudos identificaram as barreiras e dificuldades encontradas na implementação das estratégias propostas nos planos (Tabela 7) (Hrelja, 2011; Hull, 2005; López-Lambas \& Leániz, 2010; López-Lambas et al., 2012; May et al., 2003, 2008; May, 2015). As barreiras foram categorizadas e divididas 
Tabela 7 - Barreiras à implantação dos Planos de Mobilidade Urbana

\begin{tabular}{|c|c|c|c|c|c|c|c|c|c|c|c|c|}
\hline & Barreiras & 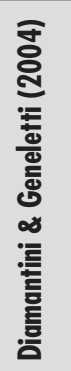 & 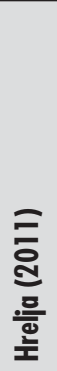 & 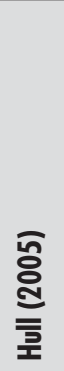 & 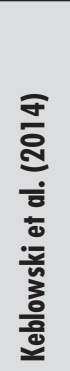 & 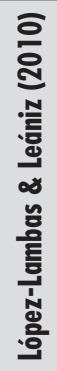 & 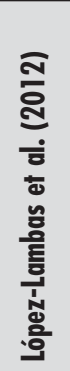 & 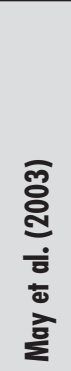 & 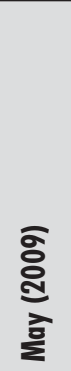 & 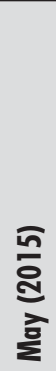 & 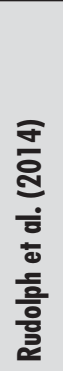 & 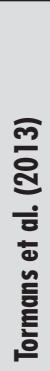 \\
\hline \multirow{21}{*}{ 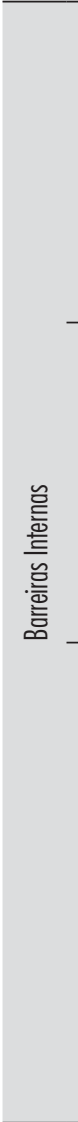 } & Entre autoridades, niveis de governo & & $\checkmark$ & $\checkmark$ & & $\checkmark$ & $\checkmark$ & $\checkmark$ & $\checkmark$ & $\checkmark$ & & \\
\hline & Entre diferentes medidas (pacotes de ações conjuntas) & & & $\checkmark$ & & & & $\checkmark$ & & $\checkmark$ & & \\
\hline & Entre medidas envolvendo diferentes modos & & & $\checkmark$ & & & & & & & & \\
\hline & Entre medidas de transporte e uso do solo & & $\checkmark$ & $\checkmark$ & & $\checkmark$ & $\checkmark$ & & & $\checkmark$ & & \\
\hline & Entre medidas de transporte e políiticas ambientais, educacação e saúde & & $\checkmark$ & $\checkmark$ & & & & $\checkmark$ & & $\checkmark$ & & \\
\hline & Entre infraestrutura, gerenciamento e tarifas & & $\checkmark$ & $\checkmark$ & & & & & & & & \\
\hline & Quantidade e diversidade de stakeholders - falta de expertise & & & & $\checkmark$ & & & & $\checkmark$ & $\checkmark$ & & $\checkmark$ \\
\hline & Compartithamento de responsabilidades & & & $\checkmark$ & & & & & $\checkmark$ & $\checkmark$ & & \\
\hline & Estabelecimento de limites espaciais - pensar o território & & & & & $\checkmark$ & & & $\checkmark$ & & & \\
\hline & Foco em uma solução específica & & & & & & & & $\checkmark$ & & $\checkmark$ & \\
\hline & Falta de recursos financeiros (preparação do plano e implementação) & & & & & $\checkmark$ & $\checkmark$ & $\checkmark$ & $\checkmark$ & $\checkmark$ & & $\checkmark$ \\
\hline & Equipe não especializada & & & & & $\checkmark$ & & & & $\checkmark$ & & $\checkmark$ \\
\hline & Número e inconsistênncias metodológicas dos indicadores & $\checkmark$ & & & & & $\checkmark$ & $\checkmark$ & $\checkmark$ & & & \\
\hline & Falta de dados & $\checkmark$ & & & & & & $\checkmark$ & $\checkmark$ & $\checkmark$ & $\checkmark$ & \\
\hline & Não entendimento dos impactos & $\checkmark$ & & & & & & $\checkmark$ & $\checkmark$ & & & \\
\hline & Problemas na modelagem & $\checkmark$ & & & & & & $\checkmark$ & $\checkmark$ & & & \\
\hline & Avaliação incompleta dos objetivos e metas & & & & & & $\checkmark$ & & $\checkmark$ & & & $\checkmark$ \\
\hline & Problemas na formaç̃õ dos custos das medidas (CBA) & & & & & & & $\checkmark$ & & & $\checkmark$ & \\
\hline & Falta de método para escolha de medidas & & & & & $\checkmark$ & & $\checkmark$ & $\checkmark$ & $\checkmark$ & & \\
\hline & Falta de projetos detalhados & & & & & & & & & $\checkmark$ & & \\
\hline & Soluçōes predominantemente tecnológicas & & & & $\checkmark$ & & & & & & & \\
\hline \multirow{12}{*}{ 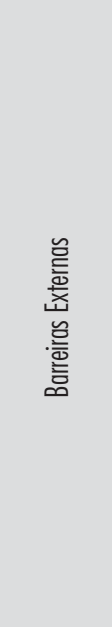 } & Inconsistência entre prioridades nacionais, regionais e locais & & $\checkmark$ & $\checkmark$ & & & & & $\checkmark$ & & & \\
\hline & Falta de controle sobre operadores de transporte coletivo & & $\checkmark$ & & & & & $\checkmark$ & & & & \\
\hline & Baixa aceitação pública das medidas & & $\checkmark$ & & $\checkmark$ & & & $\checkmark$ & & & & \\
\hline & Imediatismo na tomada de decisões, açōes de curto prazo & $\checkmark$ & $\checkmark$ & $\checkmark$ & & & & $\checkmark$ & & & & \\
\hline & Flexibilização das leis & $\checkmark$ & $\checkmark$ & $\checkmark$ & & & & & & & & \\
\hline & Privatizacão dos transportes & & $\checkmark$ & & & & & & & & & \\
\hline & Decisão política sobre técnica & $\checkmark$ & $\checkmark$ & $\checkmark$ & & $\checkmark$ & & & & & & \\
\hline & Falta de comprometimento com os princípios da sustentabilidade & $\checkmark$ & & & & & & & $\checkmark$ & & & \\
\hline & Lobby das construtoras & $\checkmark$ & & $\checkmark$ & & & & & & & & \\
\hline & Imposiç̃õo de decisões econômicas sobre as questōes ambientais & $\checkmark$ & & $\checkmark$ & & & & & & & & \\
\hline & Soluç̣ōes baseadas na mudança de comportamento das pessoas & & & $\checkmark$ & $\checkmark$ & & & & & & & \\
\hline & Falta de viscõo crítica dos transportes (problema estrutural) & & & $\checkmark$ & & & & & & & & \\
\hline
\end{tabular}

Fonte: Elaboração da autora. 
entre Aspectos Internos (Integração, Organizacionais, Técnicas) e Aspectos Externos (May et al., 2008).

May et al. (2008) descrevem o projeto Design and Implementation Support Tools for Integrated Local Land Use, Transport and the Environment (DISTILLATE), que possibilitou a identificação de barreiras através de consultas a stakehodlers. Hull (2005) ampliou os pormenores das inter-relações referentes à integração, detalhando-as em oito níveis de dificuldade, para efetivar, na prática, cada política (Tabela 8). Quanto maior o nível, maior a dificuldade de implementar.

A integração é um critério que depende da interpretação e dos interesses dos atores que representam as diferentes instâncias. Há questões que não estão resolvidas. Por exemplo: há tensões entre a implantação de ações para reduzir a dependência do automóvel e, ao mesmo tempo, garantir o crescimento econômico, incentivando as montadoras e até mesmo desenvolvendo carros elétricos. A primeira obedece aos critérios de sustentabilidade e a segunda é parte das agendas de competitividade locais.

Há um processo de implementação das ações que é simplesmente reativo e fragmentado, resultante da divisão de responsabilidades institucionais, restrições financeiras, incertezas da aceitação do público e confusão sobre a apropriação de iniciativas (Hull, 2005). As barreiras externas são erguidas por pressões dos diferentes níveis de governo e do mercado. A implementação das ações do plano, ainda que aprovadas em lei, dependem dos gestores que, muitas vezes, optam pela flexibilização dos objetivos propostos ao tomarem decisões políticas sobre as decisões técnicas (Hrelja, 2011).

\section{Métodos de avaliação dos planos}

Nove estudos avaliam os planos em dois momentos: na sua implantação e na sua elaboração. Seis estudos se detiveram na análise da implantação dos planos. López-Lambas et al. (2012) analisam os PMUs de Peterborough, Chambéry, Ferrara e Pinto e apontam problemas de integração com uso do solo, falta de financiamento para implantação do plano e falta de coordenação entre política nacional, regional e local. Diamantini \& Geneletti (2004) apontam falhas na escolha dos indicadores ambientais do plano de Trento. Rudolph et al. (2014), ao analisarem os planos de Munich, Bristol e Pitan, criticam o método custo-benefício para avaliar medidas referentes ao transporte não motorizado, por não haver dados disponíveis que quantifiquem os benefícios destas medidas.

Tabela 8 - Hierarquia dos níveis de integração

\begin{tabular}{|c|c|}
\hline Níveis de integração & Descrição \\
\hline 1. Integração física e operacional do transporte público & Integração de tarifas, horários, tickets e físicas entre as diferentes operadoras de transporte. \\
\hline 2. Integração modal & $\begin{array}{l}\text { Integração no tratamento dos diferentes modos - a pé, bicicleta, ônibus, trem, carro e aéreo - através de } \\
\text { regulamentos, taxas, critérios de avaliação e dos custos. }\end{array}$ \\
\hline 3. Integração com as necessidades do mercado & Foco nas questões de mercado quanto a eficiência, efetividade e custos do congestionamento. \\
\hline 4. Integraç̃õo com objetivos sociais & $\begin{array}{l}\text { Referente às necessidades dos diferentes grupos sociais, questões de equidade, distribuição e exclusão social. } \\
\text { Prover integraçã̃o do transporte de casa à escola, serviçcos sociais e comunitários. Atender acessibilidade } \\
\text { universal. }\end{array}$ \\
\hline 5. Integração entre políticas de transporte e questões ambientais & $\begin{array}{l}\text { Reconhecimento e incorporaç̃ão dos impactos ambiental referentes a escolha modal e políticas de transporte, } \\
\text { via modelos de avaliação, taxas e regulamentos }\end{array}$ \\
\hline 6. Integração institucional e administrativa & $\begin{array}{l}\text { Integração entre as fronteiras administrativas, identificação de políticas que produzam estratégias ótimas para } \\
\text { cada região e sub-região }\end{array}$ \\
\hline 7. Integração entre setores políiticos & $\begin{array}{l}\text { Integraç̃ão gerencial do transporte, infraestrutura, desenvolvimento urbano e proteçãa ambiental como, i.g., } \\
\text { integração e coordenação entre uso do solo e sistemas de transporte }\end{array}$ \\
\hline 8. Integração de medidas & $\begin{array}{l}\text { Reconhecimento da interdependência entre uso do solo, economia, meio ambiente, sustentabilidade e objetivos } \\
\text { sociais, como saúde, educação, affordability e inclusão. Um pacote de medidas integradas deve incluir } \\
\text { equilibrio entre fiscalizaçaca, regulamentaçã̃o e medidas soft }\end{array}$ \\
\hline
\end{tabular}

Fonte: Adaptado de Hull (2005). 
Tormans et al. (2013) usaram as teorias de Gestão de Qualidade Total (TQM) para identificar os fatores responsáveis pela baixa qualidade da política de mobilidade nos municípios da região de Flandres. Através de pesquisas de satisfação, com 25 critérios e 176 aspectos, stakeholders apontaram quatro fatores-chave para a qualidade da gestão: continuidade política, integração entre técnicos e políticos locais, perícia dos técnicos e aporte financeiro. Hrelja (2011) faz uma análise crítica da postura política das autoridades da cidade de Örebro, por terem desrespeitanto o planejado ao priorizar critérios econômicos sobre os critérios de sustentabilidade. Burggraf et al. (2014), ao apresentarem um panorama atual dos principais projetos europeus, aprofundam a necessidade de um bom sistema de indicadores e de dados consistentes para o monitoramento e para a avaliação das ações propostas no plano.

A avaliação do plano propriamente dito, definida como medida de qualidade, que leva em conta a coerência do processo que precede a implantação da política, aparece em três estudos. Wefering et al. (2013), no guia para o desenvolvimento do SUMP adotado pela CE, apresentam um check-list para cada uma das etapas de elaboração do plano e recomendam, como avaliação, que um grupo de especialistas verifique se o teor do texto cumpriu os marcos estabelecidos (Figura 4).

Kastelic et al. (2013) apresentam o projeto ADVANCE Audit, uma ferramenta de análise que identifica as forças e fraquezas do SUMP, usando o método de Gestão da Qualidade Total (TQM). Trata-se de uma série de atividades que um auditor, em conjunto com os atores locais, deve realizar, em um tempo estipulado (seis meses), para avaliar o plano. A análise gera um relatório que é analisado por um comitê independente, que irá checar a veracidade do processo e, se obtiver sucesso, a cidade recebe uma certificação.

O DFT (2004) elenca três elementos para avaliar os Local Transport Plans: qualidade do planejamento (50\%); impacto das metas (especifica padrões mínimos) (30\%) e entrega (20\%). A qualidade é avaliada em relação a seis critérios-chave e subcritérios estabelecidos. Conforme o caso, o LTP pode ser classificado como fraco, razoável, bom ou excelente. Aqueles que não contemplarem adequadamente cada subcritério são considerados falhos. Um fraco desempenho, em relação a dois ou mais subcritérios, resulta em uma avaliação fraca, para o critério como um todo. Também exige-se um desempenho regular ou bom em relação a cada critério-chave, para o plano como um todo ser considerado bom ou excelente. Os seis critérios-chave são: (i) Contexto: consistência dos objetivos, metas e projetos com a política e o planejamento; (ii) Análise dos problemas e oportunidades; (iii) Maximizar o uso dos recursos; (iv) Envolvimento dos atores; (v) Gestão da performance: qualidade do monitoramento das metas; (vi) Identificação das prioridades comuns e das questões relativas à qualidade de vida.

\section{Planos no Brasil}

À guisa de exemplificação, traz-se a situação atual dos planos de mobilidade nos municípios brasileiros. Em janeiro de 2016, o Ministério das Cidades realizou um levantamento junto aos 3.342 municípios que, até maio de 2015, deveriam ter elaborado o PMU, para concorrer a recursos financeiros federais (Brasil, 2012). Dos 2.066 respondentes, apenas 189 municípios haviam elaborado o PMU. Destes, 142 (83\%) localizam-se nas Regiões Sul e Sudeste (Brasil, 2016a) e 133 (78\%) possuem até 250 mil habitantes (Figura 5).

A baixa aderência dos municípios ao cumprimento da Lei 12.587/2012 (Brasil, 2012) incitou a proposição do Projeto de Lei 7.898/14, que prorroga, por mais três anos, até abril de 2018, o prazo final para que os municípios elaborem o PMU. A PL7.898/14, aprovada pela Câmara dos Deputados em 2015, aguarda aprovação no Senado Federal (Brasil, 2016b, 2016c). A partir do parecer da Consultoria Jurídica do MC, passou a ser exigida a aprovação do PMU por Lei Municipal (Brasil, 2016c).

A Tabela 9 relaciona 43 cidades que declararam ter elaborado o PMU, sendo que, em 29 destas (68\%), os planos foram elaborados por consultorias de engenharia ou de planejamento urbano. Importante ressaltar que alguns planos de mobilidade apontam como objetivo a elaboração de outros planos mais específicos, como o cicloviário, o de transporte coletivo, o de calçadas, o de arborização urbana, o de acessibilidade universal e o plano de iluminação pública (Edson Marchioro, 2010, 2011, 2014a, 2014b, 2015; Logit Engenharia 2014a, 2014b, 2014c, 2014d, 2014e, 2014f). Ou seja, o Plano de Mobilidade Urbana, um plano setorial, exige uma série de outros planos. A pergunta que fica é como será realizada a tão necessária integração, uma das principais barreiras ao sucesso da implementação 


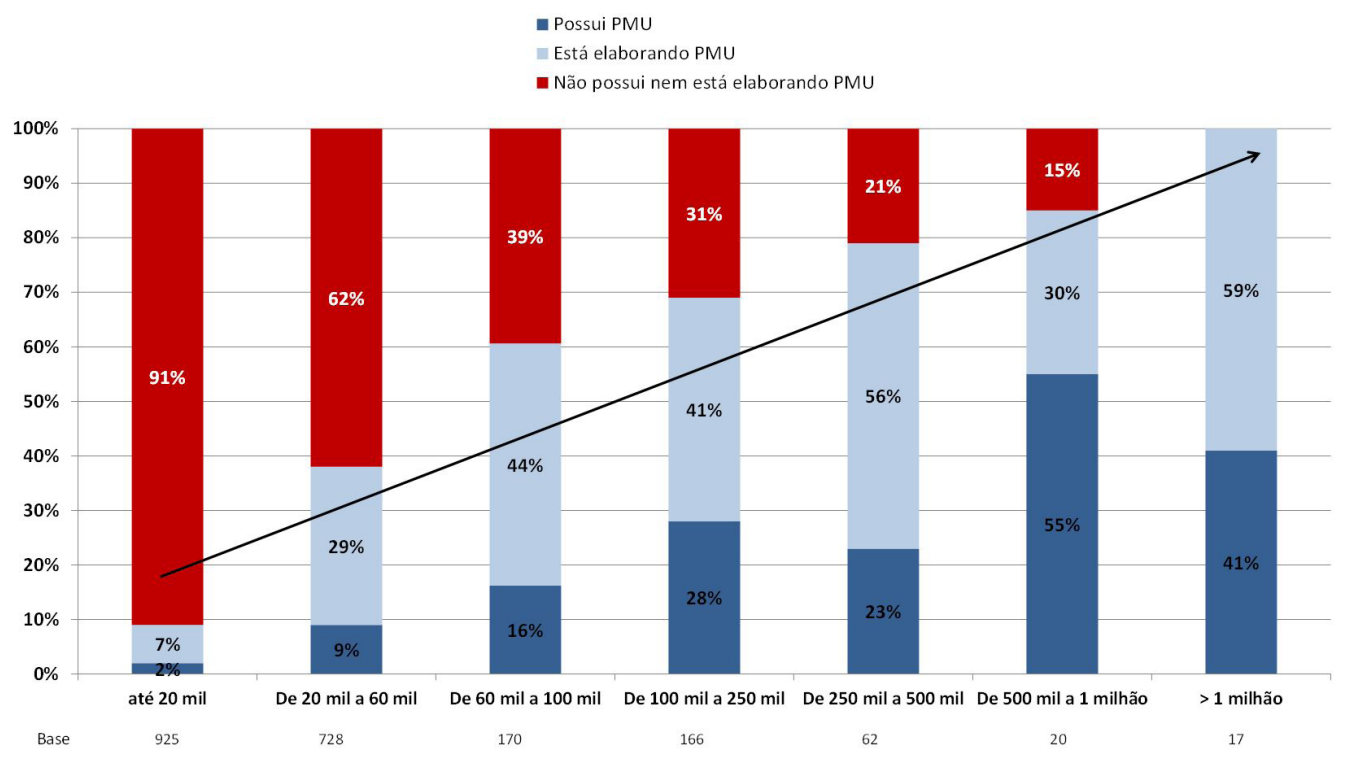

Figura 5 - Percentual dos municípios respondentes por população e relação com o PMU Fonte: Brasil (2016a).

dos planos (Hrelja, 2011; Hull, 2005; López-Lambas \& Leániz, 2010; López-Lambas et al., 2012; May et al., 2003, 2008; May, 2015).

\section{Discussão e considerações}

A revisão sistemática foi conduzida de forma a envolver os estudos e relatórios publicados com respeito à política de mobilidade urbana na Europa, por ser esta a política adotada pelo governo brasileiro (Brasil, 2013). Embora a pesquisa inicial tenha identificado cerca de 3.200 documentos, apenas 37 estudos se referiram diretamente aos planos, sendo que estes foram, em sua maioria, comunicações e relatórios sobre a política de mobilidade e as metodologias para elaboração de planos de mobilidade. Os artigos incluídos sumarizaram as barreiras apresentadas na elaboração e na efetivação dos planos de mobilidade. É importante ressaltar a importância de alinhavar as várias instâncias de integração das medidas de mobilidade, para que o plano seja um mecanismo de mudanças que garanta o direito à cidade, para todos. Sem esquecer o fato de que a mobilidade é um problema crônico e estrutural das cidades. Ao mesmo tempo em que é "motor" do desenvolvimento, é causa de externalidades negativas, que impactam diretamente na economia, na cidade e na vida das pessoas. 0 planejamento da mobilidade urbana requer a previsão de seus impactos na cidade e para as pessoas através de um aporte inter e transdisciplinar. 0 exame do problema desde uma perspectiva única é incapaz de cobrir a extensão das escalas espaciais e temporais, e tampouco dos processos envolvidos (López-Lambas \& Leániz, 2010).

A leitura dos relatórios revelou que a política de mobilidade europeia vem sendo construída desde 1996 (EC, 2001b); porém, após duas décadas de pesquisas, desenvolvimento de projetos e mais de 10 bilhões de euros investidos, observou-se que as iniciativas não foram suficientes para transformar o panorama da mobilidade. Alcançar a mobilidade sustentável requer frear a expansão urbana, reduzir os tempos e os custos dos deslcoamentos, impulsionar os modos não motorizados e o transporte coletivo. É um processo que depende do enfrentamento, pelos gestores, das pressões exercidas pela especulação imobiliária e pelos interesses econômicos, bem como da mudança e do apoio da população. As barreiras externas identificadas corroboram esta argumentação (López-Lambas et al., 2012; Hull; 2005; Keblowski et al., 2014; Hrelja, 2011; May et al., 2008).

No entanto, a política de mobilidade da Comunidade Europeia é caracterizada por leis suaves (recomendações e não obrigações), por tentar solucionar problemas através de intercâmbio de 
Tabela 9 - Relação de algumas cidades brasileiras que elaboram PMU, UF, população, Lei Municipal, responsável pela realização e fonte

\begin{tabular}{|c|c|c|c|c|c|}
\hline Municipio & UF & População & Lei & Realizaç̃̃o & Fonte \\
\hline Almirante Tamandaré & $P R$ & 103.245 & $39 / 2015$ & Órgão público & Pref. Mun. de Almirante Tamandaré (2015) \\
\hline Anchieta & ES & 23.894 & & Consultoria & Logit Engenharia (2014a) \\
\hline Aracruz & ES & 81.746 & $3.915 / 2015$ & Consultoria & Logit Engenharia (2014b) \\
\hline Bagé & RS & 116.792 & & Consultoria & Prefeitura Municipal de Bagé (2015) \\
\hline Belo Horizonte & MG & 1.433 .000 & $15.317 / 2013$ & Órgão público & Prefeitura Municipal de Belo Horizonte (2010) \\
\hline Cachoeira de Itapemirim & ES & 189.889 & $7.180 / 2015$ & Consultoria & Logit Engenharia (2014c) \\
\hline Campo Grande & MS & 787.204 & $12.681 / 2015$ & Consultoria & Logitrans (2015) \\
\hline Caraguatatuba & SP & 100.899 & $2.241 / 2015$ & Órgão público & Caraguatatuba (2015) \\
\hline Catanduva & $S P$ & 112.843 & & Consultoria & BID (2015) \\
\hline Chapecó & SC & 183.561 & & Órgão público & FEPESE (2015) \\
\hline Colatina & ES & 111.788 & & Consultoria & Logit Engenharia (2014d) \\
\hline Concórdia & SC & 68.627 & $4.845 / 2016$ & Consultoria & Via 11 (2016) \\
\hline Dourados & MS & 173.647 & & Consultoria & Edson Marchioro (2015) \\
\hline Farroupilha (RT) & RS & 71.281 & & Consultoria & Edson Marchioro (2014a) \\
\hline Fortaleza & CE & 862.750 & & Órgão público & IPANFOR (2015) \\
\hline Gaspar & SC & 57.958 & $65 / 2015$ & Órgão público & Gaspar (2015) \\
\hline Guarapari & ES & 100.528 & & Consultoria & Logit Engenharia (2014g) \\
\hline Ipojuca & $\mathrm{PE}$ & 80.542 & & Consultoria & CL Engenharia e Urbanismo (CL, 2015) \\
\hline Itanhaém & $\mathrm{SP}$ & 87.053 & & Consultoria & Logit Engenharia (2014e) \\
\hline Joinville & SC & 486.803 & & Órgão público & Prefeitura Municipal de Joinville (2015) \\
\hline Linhares & ES & 109.653 & & Consultoria & Logit Engenharia (2014f) \\
\hline Manaus & AM & 1.802 .525 & $2.074 / 2015$ & Consultoria & Oficina Engenheiros Consultores Associados (2015) \\
\hline Monte Alto & SP & 46.647 & $395 / 2015$ & Consultoria & Genos Consultoria Ambiental (2015) \\
\hline Olímpia & $\mathrm{SP}$ & 50.024 & & Órgão público & Prefeitura Municipal de Olímpia (2015) \\
\hline Osasco & $S P$ & 666.469 & & Consultoria & Oficina Engenheiros Consultores Associados (2016) \\
\hline Paranaguá & PR & 140.450 & & Órgão público & Prefeitura Municipal de Paranaguá (2016) \\
\hline Pindamonhangaba & $S P$ & 147.034 & $51 / 2015$ & Órgão público & Pindamonhangaba (2015) \\
\hline Porto Alegre & RS & 1.409 .939 & & Órgão público & Prefeitura Municipal de Porto Alegre (2016) \\
\hline Presidente Prudente & SP & 207.625 & $8.970 / 2015$ & Consultoria & Edson Marchioro (2014b) \\
\hline Ribeirão Preto & $\mathrm{SP}$ & 605.114 & & Consultoria & Oficina Engenheiros Consultores Associados (2012) \\
\hline Rio das Ostras & RJ & 105.757 & & Órgão público & Prefeitura Municipal de Rio das Ostras (2015) \\
\hline Santa Maria & RS & 261.031 & $98 / 2015$ & Consultoria & Idom Consulting (2013) \\
\hline Santa Rosa & RS & 72.240 & & Consultoria & Edson Marchioro (2010) \\
\hline Santos & $\mathrm{SP}$ & 419.086 & & Órgão público & Prefeitura Municipal de Santos (2015) \\
\hline São José do Rio Preto & SP & 408.435 & $11.736 / 2015$ & Consultoria & São José do Rio Preto (2015) \\
\hline São Jose dos Campos & $\mathrm{SP}$ & 629.921 & $576 / 2016$ & Órgão público & Prefeitura Municipal de São José dos Campos (2015) \\
\hline São Luis Gonzaga & RS & 34.556 & $5.494 / 2015$ & Consultoria & Urbana Logística Ambiental do Brasil Ltda. (2015) \\
\hline São Paulo & $\mathrm{SP}$ & 11.253 .503 & & Órgão público & Prefeitura Municipal de São Paulo (2015) \\
\hline Sapiranga & RS & 74.985 & $5.749 / 2015$ & Consultoria & Alto Uruguai (2016) \\
\hline Sorocaba & SP & 586.311 & & Consultoria & Urbes Trânsito e Transportes (2014) \\
\hline Tremembé & SP & 40.984 & $299 / 2016$ & Órgão público & IPPLAN (2015) \\
\hline Uberlândia & MG & 584.102 & & Órgão público & Prefeitura Municipal de Uberlândia (2010) \\
\hline União da Vitória & PR & 52.753 & $4.526 / 2015$ & Órgão público & União da Vitória (2015) \\
\hline Vacaria & RS & 61.345 & & Consultoria & Edson Marchioro (2011) \\
\hline Vargem Grande Paulista & $\mathrm{SP}$ & 42.946 & & Órgão público & Prefeitura Municipal de Vargem Grande Paulista (2016) \\
\hline Venâncio Aires & RS & 65.964 & $077 / 2014$ & Órgão público & Venôncio Aires (2014) \\
\hline
\end{tabular}

Fonte: Elaboração da autora. 
melhores práticas e transferência de conhecimento (Halpern, 2014). A introdução do PMUS empodera a autoridade local e confirma a dimensão urbana (territorial) da mobilidade, em oposição a uma abordagem setorial (transporte) (Halpern, 2014). O Plano de Mobilidade Urbana Sustentável é um plano estratégico participativo para o futuro da cidade e deve estar estruturado por objetivos, metas, indicadores e ações. Essa metodologia, recomendada pela Comunidade Europeia para os países europeus, também foi adotada em outros países, como Brasil, México e Índia (Böhler-Baedeker et al., 2014).

0 pensamento único que orienta o planejamento da mobilidade parece ir de encontro à representação da cidade global, competitiva e sustentável (Keblowski et al., 2014), por não elencar os conflitos sociopolíticos da estrutura urbana. 0 viés crítico, levantado por Keblowski et al. (2014) e Baeten (2000), é um contraponto importante, que deve ser arrolado nas propostas de planejamento, ainda mais quando se trata de países em vias de desenvolvimento. As barreiras externas identificadas - a flexibilização dos objetivos, a prevalência de decisões políticas e econômicas, o lobby imobiliário - sinalizam que dificilmente haverá mudanças significativas enquanto as iniquidades que sustentam a problemática urbana, do transporte e da mobilidade não forem enfrentadas.

As barreiras internas estão relacionadas a problemas na integração, na organização e nos aspectos técnicos, aspectos ligados à etapa metodológica de elaboração do plano. Entre as causas, ressalta-se a falta de equipes especializadas, a falta de dados, número de indicadores e, deficiências na identificação dos impactos das medidas propostas (Tabela 10). Ao proceder a avaliação do plano, e aqui se retorna à definição de avaliação como uma medida de qualidade de acordo com a coerência do processo que precede a implantação (Kastelic et al., 2013; DFT, 2004; Tormans et al., 2013), teoricamente, algumas dessas deficiências deveriam ser apontadas para não comprometer a qualidade do plano.

A avaliação proposta pelo guia de elaboração do PMUS (Wefering et al., 2013), é de um check-list como garantia de que cada etapa da elaboração apresente o mínimo recomendado. Já o DFT (2004) avalia a qualidade do plano através de critérios e subcritérios estabelecidos, que irão classificar cada LTP como fraco, razoável, bom ou excelente. Salienta-se a ausência de estudos sobre os resultados dessas avaliações.

Por outro lado, trazendo essa discussão para a realidade das cidades brasileiras, que é, ao fim e ao cabo, o objetivo desta investigação - identificar como se constituem os planos e como é realizada sua avaliação —, inferiu-se a necessidade de identificar, clara e resumidamente, o que é solicitado pelos guias: i) se os objetivos propostos satisfazem as necessidades apontadas; ii) se as metas são factíveis; iii) se os indicadores são adequados; iv) se as ações contemplam os objetivos; v) se há viabilidade financeira, e vi) se há definição de quem será o gestor de cada ação e das formas de integração com as outras esferas de governo, principalmente nos municípios que fazem parte de regiões metropolitanas. Enfim, estabelecer um resumo sistemático que possibilite avaliar a consistência do plano no que ele se propõe. Seria interessante elaborar uma sistemática de avaliação das três etapas consideradas fundamentais para a elaboração do plano: Participação (como foram organizados os métodos de participação), Diagnóstico (as necessidades elencadas pelos atores, coletas de dados e levantamentos) e as Ações (fundamentos, cenários, avaliação de impactos, custos, financiamento, controle e gestão). Também seria importante sistematizar/compilar as normas, os critérios e os padrões existentes para as ações mínimas exigidas pela Lei 12.587 (Brasil, 2012), como, por exemplo: dimensões mínimas de ciclovias, calçadas, padrões de acessibilidade, etc.). Porém, fundamentalmente, as ações propostas devem responder às perguntas: Qual é o público-alvo da ação? Quem será beneficiado? As propostas devem objetivar uma mudança social positiva, a melhoria da qualidade de vida e o aumento da justiça social, no espaço urbano.

\section{Referências}

Almirante Tamandaré. Prefeitura Municipal. (2015). Plano Diretor de Mobilidade Urbana. Recuperado em novembro de 2016, de http://tamandare.pr.gov.br/admin/files/paginas/ arquivos/046acce7457ca06f81140aa813aab7f1.pdf 
Tabela 10 - Cruzamento entre os passos propostos para elaboração do SUMP e as barreiras identificadas

\begin{tabular}{|c|c|c|}
\hline Etapas & Passos de elaboração SUMP & Barreiras internas identificadas \\
\hline \multirow{11}{*}{ 1. Preparação } & $\begin{array}{l}1.1 \text { Comprometimento com os princípios da } \\
\text { sustentabilidade }\end{array}$ & Falta de integração entre autoridades, níveis de governo; \\
\hline & 1.2 Avaliar impactos regional/nacional & Não entendimento dos impactos; Estabelecimento de limites espaciais \\
\hline & 1.3 Realizar diagnóstico & Falta de dados; Avaliação incompleta dos objetivos; \\
\hline & 1.4 Avaliar a disponibilidade de recursos & Falta de recursos financeiros (preparo e implementação) \\
\hline & 1.6 Identificar atores-chave & Quantidade e diversidade de stakeholders $\bigotimes$ falta de expertise \\
\hline & 2.1 Olhar para além dos limites e responsabilidades & Compartilhamento de responsabilidades; Estabelecimento de limites espaciais - pensar o território \\
\hline & $\begin{array}{l}2.2 \text { Buscar coordenação política e abordagem } \\
\text { integrada }\end{array}$ & $\begin{array}{l}\text { Integração entre níveis de governo; Entre diferentes medidas; Entre medidas envolvendo diferentes modos; } \\
\text { Entre medidas de transporte e uso do solo, políticas ambientais, educação e saúde; Entre infraestrutura, } \\
\text { gerenciamento e tarifas; Estabelecimento de limites espaciais }\end{array}$ \\
\hline & 2.3 Envolver atores e cidadãos no processo & Quantidade e diversidade de stakeholders - falta de expertise \\
\hline & $\begin{array}{l}2.4 \text { Estabelecer plano de trabalho e mecanismos } \\
\text { de gestão }\end{array}$ & Compartihamento de responsabilidades \\
\hline & $\begin{array}{l}\text { 3.1 Preparar uma análise dos problemas e } \\
\text { oportunidades }\end{array}$ & Inconsistência entre avaliação e metas \\
\hline & 3.2 Desenvolver cenários & Falta de dados; Não entendimento dos impactos; Problemas na modelagem \\
\hline \multirow{7}{*}{ 2. Escolha dos objetivos } & $\begin{array}{l}\text { 4.1 Desenvolver uma visão comum da mobilidade } \\
\text { e da cidade }\end{array}$ & Avaliação incompleta dos objetivos \\
\hline & 5.1 Identificar prioridades para a mobilidade & Não entendimento dos impactos; Avaliação incompleta dos objetivos \\
\hline & 5.2 Desenvolver metas inteligentes & Soluç̃oes predominantemente tecnológicas \\
\hline & 6.1 Identificar quais são as medidas mais efetivas & $\begin{array}{l}\text { Integração entre níveis de governo; Entre diferentes medidas; Entre diferentes modos; Entre medidas de } \\
\text { transporte e uso do solo, políticas ambientais, educacacão e saúde; Entre infraestrutura, gerenciamento e } \\
\text { tarifas; Avaliação incompleta dos objetivos; Falta de evidência da performance de soluçōes específicas; Falta } \\
\text { de método para escolha de medidas }\end{array}$ \\
\hline & 6.2 Aprender com a experiência de outras cidades & Falta de evidência da performance de soluções especificas \\
\hline & 6.3 Considerar melhor custo-beneficio & Falha na avaliação financeira das medidas (CBA) \\
\hline & $\begin{array}{l}6.4 \text { Usar sinergia para criar um pacote de medidas } \\
\text { integradas }\end{array}$ & $\begin{array}{l}\text { Falta de método para escolha de medidas; Foco em uma solução específica; Soluç̃ões predominantemente } \\
\text { tecnológicas }\end{array}$ \\
\hline \multirow{3}{*}{ 3. Elaboração } & $\begin{array}{l}\text { 7.1 Compartilhamento de responsabilidades; Atribuir } \\
\text { responsabilidades e alocar recursos }\end{array}$ & Falta de recursos financeiros (preparo e implementação) \\
\hline & 7.2 Preparar plano de ação orçado & Falta de projetos detalhados; Falta de recursos financeiros (preparo e implementaç̃ó) \\
\hline & 8.1 Providenciar monitoramento e avaliação & Número e inconsistências metodológicas dos indicadores; Falta de dados \\
\hline 4. Implementação & $\begin{array}{l}10.3 \text { Conferir o progresso das metas em relaçãa } \\
\text { aos objetivos }\end{array}$ & Número e inconsistências metodológicas dos indicadores; Avaliação incompleta dos objetivos \\
\hline
\end{tabular}

Fonte: Elaboração própria.

Alto Uruguai. Engenharia e Gestão de Cidades. (2016). Plano Diretor de Mobilidade Urbana Sapiranga-RS Plano de Ações. Concórdia. Recuperado em novembro de 2016, de http:// www.sapiranga.rs.gov.br/site/servico/visualizar/idDep/1/ id/54/?PlanMob-Sapiranga.html

Associação Nacional das empresas de Transportes Urbanos NTU. (2015, jan/fev). Menos de 30\% das principais cidades brasileiras possuem plano de mobilidade urbana. Revista NTUrbano, 3(13), 4-34.

Baeten, G. (2000). The tragedy of the highway: empowerment, disempowerment and the politics of sustainability discourses and practices. European Planning Studies, 8(1), 69-86. http:// dx.doi.org/10.1080/096543100110938.
Bagé. Prefeitura Municipal. (2015). Plano diretor de transporte e mobilidade urbana. Consultoria Prócidades. Relatório 3.

Banco Interamericano de Desenvolvimento - BID. (2015). Plano Diretor de Mobilidade Catanduva. Catanduva: Idom, Tectran. Recuperado em novembro de 2016, de http://www. catanduva.sp.gov.br/DynamicFiles/File/PDMUCatanduva/ Produto_5_6_8/Etapa\%203.1.\%203.2\%20e\%203.4_12.\%20 Proposta\%20para\%20o\%20PDMU.pdf

Belo Horizonte. Prefeitura Municipal. (2010). Plano de Mobilidade Urbana de Belo Horizonte. BHTrans. Logit Engenharia: desenvolvido entre 2007-2010. Relatório final. Recuperado em novembro de 2016, de http://www.bhtrans. 
pbh.gov.br/portal/pls/portal/!PORTAL.wwpob_page. show?_docname=9604263.PDF

Böhler-Baedeker, S., Kost, C., \& Merforth, M. (2014). Urban mobility plans: national approaches and local practice: sustainable urban transport (Technical Document, No. 13). Germany: Deutsche Gesellschaft für Internationale Zusammenarbeit.

Brasil. (2012, 3 de janeiro). Lei no 12.587, de 3 de janeiro de 2012. Dispõe sobre as diretrizes da Política Nacional de Mobilidade Urbana. Brasília: Diário Oficial da União. Recuperado em maio de 2015, de www.planalto.gov.br/ ccivil_03/_ato2011-2014/2012/lei/112587.htm

Brasil. Ministério das Cidades. (2013). Planejamento em mobilidade urbana: projeto diálogos setoriais. Brasília: MC.

Brasil. (2015). Caderno de referência para elaboração de plano de mobilidade urbana. Brasília: MC.

Brasil. Ministério das Cidades. (2016a). Levantamento sobre a situação dos Planos de Mobilidade Urbana nos municípios brasileiros. Brasília: MC. Recuperado em novembro de 2016, de http://www.cidades.gov.br/mobilidade-urbana/ planejamento-da-mobilidade-urbana/

Brasil. Ministério das Cidades. (2016b). Perguntas frequentes: o Plano de Mobilidade Urbana precisa ser aprovado por lei municipal? Brasília: MC. Recuperado em novembro de 2016, de http://www.cidades.gov.br/mobilidade-urbana/ perguntas-frequentes-semob

Brasil. Câmara dos Deputados. (2016c). Câmara prorroga o prazo para os municípios elaborarem o Plano de Mobilidade Urbana. Brasília. Recuperado em novembro de 2016, de http://www.camara.gov.br/proposicoesWeb/fichadetram itacao?idProposicao $=621861$

Browne, D., \& Ryan, L. (2011). Comparative analysis of evaluation techniques for transport policies. Environmental Impact Assessment Review, 31(3), 226-233. http://dx.doi. org/10.1016/j.eiar.2010.11.001.

Burggraf, K., Gühnemann, A., \& May, A. D. (2014). Why is monitoring and evalution a challenge in sustainable urban mobility planning? Ch4allenge. 12 p. Recuperado em maio de 2015, de http://www.eltis.org/sites/eltis/files/tool/ challenge_description_monitoring_evaluation_final.pdf

Caraguatatuba. (2015, 3 de julho). Lei $n^{\circ} 2.241$, de 3 de julho de 2015. Institui o Plano Municipal de Mobilidade Urbana do Município de Caraguatatuba. Caraguatatuba: PlanMob Caraguá. Recuperado em novembro de 2016, de http:// www.legislacaocompilada.com.br/caraguatatuba/Arquivo/ Documents/legislacao/html/L22412015.html
Cunha Lanfermann Engenharia e Urbanismo Ltda - CL. (2015). Plano de Mobilidade Urbana do Município de Ipojuca. Ipojuca. Recuperado em novembro de 2016, de http://www.ipojuca.pe.gov.br/

Department For Transport - DFT. (2004). Full guidance on local transport plans (2. ed.). UK: Department for Transport. 106 p. Recuperado em maio de 2015, de http://www. polisnetwork.eu/uploads/Modules/PublicDocuments/ Guidance\%20on\%20LTP.pdf

Diamantini, C., \& Geneletti, D. (2004). Reviewing the application of SEA to sectoral plans in Italy: the case of the mobility plan of Alpine region. European Environment, 14(2), 123-133. http://dx.doi.org/10.1002/eet.341.

Dziekan, K., Riedel, V., Abraham, M., Kettner, S., Daubitz, S., \& Muller, S. (2013). Evaluation matters: a practitioner's guide to sound evaluation for urban mobility measures. Berlin: Waxmann.

Edson Marchioro. (2010). Plano Diretor de Mobilidade Urbana de Santa Rosa. Recuperado em novembro de 2016, de http:// www.santarosa.rs.gov.br/downloads_ver.php?dow_id=592

Edson Marchioro. (2011). Plano Diretor de Mobilidade Urbana e Plano Viário de Vacaria. Recuperado em novembro de 2016, de http://www.vacaria.rs.gov.br/docs/mobilidade/ AUDIENCIA-PUBLICA-10-11-2011.pdf

Edson Marchioro. (2014a). Plano Diretor de Mobilidade Urbana de Farroupilha. Relatório Técnico. Recuperado em novembro de 2016, de http://farroupilha.rs.gov.br/ novo/download/mobilidade_/RELAT\%C3\%93RIO\%20 T\%C3\%89CNICO\%2001\%20FARROUPILHA.pdf

Edson Marchioro. (2014b). Plano diretor de mobilidade urbana de Presidente Prudente. Relatório Técnico 01. Recuperado em novembro de 2016, de http://www.presidenteprudente. sp.gov.br/outros/mobilidade/default.asp.pdf

Edson Marchioro. (2015). Arquitetura, urbanismo e engenharia S/S. Plano Diretor de Mobilidade Urbana de Dourados - MS. Relatório Técnico. Recuperado em novembro de 2016, de http://www.dourados.ms.gov.br/wpcontent/ uploads/2016/03/DOU-RELATORIO-TEC-ETAPA02-v10.pdf

Eltis. (2016). The urban mobility observatory. Recuperado em novembro de 2016, de www.eltis.org

European Cities and Regions Networking from Innovative Transport Solutions - POLIS. (2016). Recuperado em novembro de 2016, de www.polisnetwork.eu

European Comission - EC. (2001a). Urban transport: thematic synthesis of transport research results (Vol. 7). Paper 5 of 
10. Extra project (final). 99 p. Recuperado em maio de 2015, de http://www.ocs.polito.it/biblioteca/mobilita/ UrbanTransportEXTRA.pdf

European Comission - EC. (2001b). White paper: European transport policy for 2010: time to decide. Luxembourg: Office for Official Publications of the European Communities. $126 \mathrm{p}$.

European Comission - EC. (2004). SMILE: Sustainable Mobility Initiatives for Local Environment: towards sustainable urban transport policies: recommendations for local authorities. Spain. 68 p.

European Comission - EC. (2007a). Sustainable urban transport plans. Annex. Luxembourg: Office for Official Publications of the European Communities. 122 p.

European Comission - EC. (2007b). Green paper: towards a new culture for urban mobility. Brussels. COM(2007)551 final. $24 \mathrm{p}$.

European Comission - EC. (2007c). Sustainable urban transport plans: preparatory document in relation to the follow-up of thematic strategy on the urban environment. Luxembourg: Office for Official Publications of the European Communities. 20 p. Main Document.

European Comission - EC. (2009). Action plan on urban mobility: communication from the commission to the european parliament, the council, the european economic and social committee and the committee of the regions. $\mathrm{COM}(2009)$ 490 final. Brussels. 15 p.

European Comission - EC. (2011). Impact assessment. White paper: roadmap to a single european transport area: towards a competitive and resource efficient transport system. Brussels. 71 p. Commissiona staff working paper.SEC(2011) 358 final.

European Comission - EC. (2012). Action plan on urban mobility: state of play: directorate-general for mobility and transport: directorate C: innovative and sustainable mobility. Brussels. 15 p.

European Comission - EC. (2013a). A concept for a sustainable urban mobility plans. Brussels. COM(2013) 913 final. Annex 1. $5 \mathrm{p}$.

European Comission - EC. (2013b). Together towards competitive and resource-efficient urban mobility. Communication from the Commission to the European parliament, the council, the European economic and social committee and the committee of the regions. Brussels. 12 p. COM (2013) 913 final.

European Comission - EC. (2013c). Guidelines: developing and Implementing a Sustainable Urban Mobility Plan. Brussels. $151 \mathrm{p}$.
Eurostat. (2016). Europe 2020 indicators: climate change and energy: statistics explained. Recuperado em abril de 2016, de http://ec.europa.eu/eurostat/statistics-explained/

Figueiredo, M. F., \& Figueiredo, A. M. C. (1986). Avaliação política e avaliação de políticas: um quadro de referência teórica. Belo Horizonte.

Fundação de Estudos e Pesquisas Socioneconômicos FEPESE. (2015). Estudo de mobilidade urbana de Chapecó. Relatório final. Recuperado em novembro de 2016, de https://web.chapeco.sc.gov.br/documentos/?f=/Documentos/ Desenvolvimento\%20Urbano/Estudo\%20Mobilidade\%20 Urbana.pdf

Gaspar. (2015, 23 de novembro). Lei Complementar $n^{\underline{o}}$ 65, de 23 de novembro de 2015. Institui o Plano Diretor de Transporte e Mobilidade Urbana de Gaspar. Recuperado em novembro de 2016, de http://www.gaspar.sc.gov.br/cms/ pagina/ver/codMapaItem/71012

Genos Consultoria Ambiental. (2015). Plano de Mobilidade Urbana de Monte Alto/SP. Relatório 2. Recuperado em novembro de 2016, de http://montealto.sp.gov.br/site/ planos-municipais/

Gil, A., Calado, H., \& Bentz, J. (2011). Public participation in municipal transport planning processes: the case of the sustainable mobility plan of Ponta Delgada, Azores, Portugal. Journal of Transport Geography, 19(6), 1309-1319. http:// dx.doi.org/10.1016/j.jtrangeo.2011.06.010.

Halpern, C. (2014). Urban mobility: what role for the European Union? Explaining dynamics of European Union policy design since 1995. European Planning Studies, 22(12), 2526-2541. http://dx.doi.org/10.1080/09654313.2013.844775.

Hrelja, R. (2011). The tyranny of small decisions: unsustainable cities and local day-to-day transport planning. Planning Theory \& Practice, 12(4), 511-524. http://dx.doi.org/10.1 080/14649357.2011.626312.

Hull, A. (2005). Integrated transport planning in the UK: from concept to reality. Journal of Transport Geography, 13(4), 318-328. http://dx.doi.org/10.1016/j.jtrangeo.2004.12.002.

Idom Consulting. (2013). Plano diretor de mobilidade urbana: propostas de atuação. Relatório Técnico III. Recuperado em novembro de 2016, de http://iplan.santamaria.rs.gov.br/ site/projeto/visualizar/id/62

Instituto de Pesquisa Administração e Planejamento - IPPLAN. (2015). Plano de mobilidade urbana de Tremembé: construindo o futuro das cidades para as pessoas. Relatório final da mobilidade urbana. Recuperado em novembro de 2016, de http:// 
www.tremembe.sp.gov.br/wp-content/uploads/2016/05/ RelDiagnosticoPrognosticoPlanoMobilidadeUrbana.pdf

Instituto de Pesquisa e Economia Aplicada - IPEA. (2011). A nova lei de diretrizes da política nacional de mobilidade urbana (Comunicados do IPEA, No. 128). Brasília.

Instituto de Planejamento de Fortaleza - IPANFOR. (2015). Plano de Mobilidade de Fortaleza. PlanMob. Recuperado em novembro de 2015, de http://fortaleza2040.fortaleza.ce.gov. br/site/assets/files/publications/fortaleza2040_plano_de_ mobilidade_urbana_17-08-2015.pdf

Joinville. Prefeitura Municipal. (2015). Plano Municipal de Mobilidade Urbana: PlanMob 2015. Recuperado em novembro de 2016, de https://ippuj.joinville.sc.gov.br

Kastelic, J., Duportail, V., \& Meerschaert, V. (2013). Final advance auditscheme and guidelines: advance, better planning, better cities. Austria: Austrian Mobility Research. 74 p. Recuperado em maio de 2015, de https://ec.europa. eu/energy/intelligent/projects/sites/iee-projects/files/ projects/documents/advance_audit_scheme_en.pdf

Keblowski, W., Bassens, D., \& Criekingen, M. V. (2014). The differential performativity of academic knowledges in urban transport and mobility policy and practice: a view from Brussels. Brussels: Cosmopolis Centre for Urban Research.

Lindenau, M., Tovaas, K., \& Wefering, F. (2014). Guidelines for policy-makers: policy integration, policy processes and participation in sustainable urban mobility planning. Rupprecht Consult. SHAPE-IT Policy Guidelines for PolicyMakers. Recuperado em maio de 2015, de http://www. rupprecht-consult.eu/uploads/tx_rupprecht/SHAPEIT_Policy-Guidelines_RC-final.pdf

Logit Engenharia. (2014a). Plano de Mobilidade do Município de Anchieta. Recuperado em novembro de 2016, de http:// anchieta.planodemobilidade.com.br/

Logit Engenharia. (2014b). Plano de Mobilidade do Município de Aracruz. Recuperado em novembro de 2016, de http:// aracruz.planodemobilidade.com.br/

Logit Engenharia. (2014c). Plano de Mobilidade do Município de Cachoeira do Itapemirim. Recuperado em novembro de 2016, de http://cachoeiradeitapemirim.planodemobilidade. com.br/

Logit Engenharia. (2014d). Plano de Mobilidade do Município de Colatina. Recuperado em novembro de 2016, de http:// colatina.planodemobilidade.com.br/
Logit Engenharia. (2014e). Plano de Mobilidade do Município de Itanhaém. Recuperado em novembro de 2016, de http:// itanhaem.planodemobilidade.com.br/

Logit Engenharia. (2014f). Plano de Mobilidade do Município de Linhares. Recuperado em novembro de 2016, de http:// linhares.planodemobilidade.com.br/

Logit Engenharia. (2014g). Plano de Mobilidade do Município de Guarapari. Recuperado em novembro de 2016, de https:// sedurb.es.gov.br/planos-de-mobilidade-urbana

Logitrans. Logística, Engenharia e Transporte. (2015). Plano diretor de transporte e mobilidade urbana de Campo Grande. Recuperado em novembro de 2016, de http://docslide.com. br/documents/logitrans-logistica-engenharia-e-transporteplano-diretor-de-transporte.html

López-Lambas, M. E., \& Leániz, C. L.-G. (2010). The Spanish territorial context: an analysis in light of the European Urban Sustainable Mobility Plans. In Proceedings of the 12th World Conference on Transport Research (pp. 1-19). Lisbon, Portugal.

López-Lambas, M.E., Corazza, M. V., Monzon, A., \& Musso, A. (2010). Urban mobility plans throughout Europe: a definitive challenge towards sustainability. In Proceedings of the TRB 89th Annual Meeting (pp. 16). Washington.

López-Lambas, M. E., Corazza, M. V., Monzon, A., \& Musso, A. (2012). Rebalancing urban mobility: a tale of four cities. ICE Virtual Library, 166(DP5), 274-287.

Lopez-Ruiz, H., Christidis, P., Demirel, H., \& Kompil, M. (2013). Quantifying the effects of sustainable urban mobility. Luxembourg: Office of the European Union. JRC technical reports.

May, A. D. (2003). Developing sustainable urban land use and transport strategies: a decision makers' guidebook: prospects: procedures for recommending optimal sustainable planning of European City Transport Systems (Deliverable, no. 15). Noruega: Institute of Transport Economics. $52 \mathrm{p}$.

May, A. D. (2009). Improving decision-making for sustainable urban transport: an introduction to the distillate research programme. European Journal of Transport and Infrastructure Research, 9(3), 184-201.

May, A. D. (2015). Encouraging good practice in the development of Sustainable Urban Mobility Plans. World Conference on Transport Research Society. Case Studies on Transport Policy, 3(1), 3-11. 
May, A. D., Jopson, A. F., \& Matthews, B. (2003). Research challenges in urban transport policy. Transport Policy, 10(3), 157-164. http://dx.doi.org/10.1016/S0967-070X(03)00039-8.

May, A. D., Kelly, C., Shepherd, S., \& Jopson, A. (2012). An option generation tool for potential urban transport policy packages. Transport Policy, 20, 162-173. http://dx.doi. org/10.1016/j.tranpol.2012.01.012.

May, A. D., Page, M., \& Hull, A. (2008). Developing a set of decision-support tools for sustainable urban transport in the UK. Transport Policy, 15(6), 328-340. http://dx.doi. org/10.1016/j.tranpol.2008.12.010.

May, A. D., Kelly, C., \& Shepherd, S. (2006). The principles of integration in urban transport strategies. Transport Policy, 13(4), 319-327. http://dx.doi.org/10.1016/j. tranpol.2005.12.005.

May, T., Jarvy-Nykanen, T., Minken, H., Ramjerdi, F., Matthews, B., \& Monzón, A. (2001). Cities' decision-making requirements prospects: procedures for recommending optimal sustainable planning of European city transport systems (Deliverable, No. 1). Leeds: Institute for Transport Studies, University of Leeds. $82 \mathrm{p}$.

Minken, H., Jonsson, D., Shepherd, S., Järvi, T., May, T., Page, M., Pearman, A., Pfaffenbichler, P., Timms, P., \& Vold, A. (2003). Developing sustainable urban land use and transport strategies: a methodological guidebook: prospects. procedures for recommending optimal sustainable planning of european city transport systems (Deliverable, No. 14). Noruega: Institute of Transport Economics. $224 \mathrm{p}$.

Mobilize. Mobilidade Urbana Sustentável Brasil. (2011). Estudo Mobilize 2011: diagnóstico da mobilidade urbana sustentável em capitais brasileiras. Portal Mobilize. Recuperado em maio de 2015, de http://www.mobilize.org.br/estudos/10/ estudo-mobilize-2011-diagnostico-da-mobilidade-urbanasustentavel-em-capitais-brasileiras.html

Oficina Engenheiros Consultores Associados. (2012). Plano de Mobilidade Urbana de Ribeirão Preto. Recuperado em novembro de 2016, de http://www.ribeiraopreto.sp.gov. br/transerp/mobilidade/i07mobilidade.php

Oficina Engenheiros Consultores Associados. (2015). Plano de mobilidade urbana de Manaus (Vol. 1). Recuperado em novembro de 2016, de http://smtu.manaus.am.gov.br/ planmob/

Oficina Engenheiros Consultores Associados. (2016). Plano de Mobilidade Urbana de Osasco. Recuperado em novembro de 2016, de http://www.seplag.osasco.sp.gov.br/Content/ uploads/publicacao/arquivo/d36ab199-8d5d-4f05-8886ad0caaab5910.pdf/
Olímpia. Prefeitura Municipal. (2015). Proposta para o Plano de Mobilidade Urbana de Olimpia 2015. PRODEM Desenvolvimento Municipal. Recuperado em novembro de 2016, de http://www.olimpia.sp.gov.br/dowloads/outros/ planomobilidade/plano-de-mobilidade-urbana.pdf

Oliveira, J. A., Jr. (2012). Princípios, diretrizes e objetivos da Lei 12.587/2012: por um pacto social em prol da mobilidade urbana. Revista UFG, 12, 18-27.

Paranaguá. Prefeitura Municipal. (2016). Plano de mobilidade urbana município Paranaguá. Recuperado em novembro de 2016, de http://www.paranagua.pr.gov.br/imgbank2/file/ Plano\%20de\%20Mobilidade\%20Munic\%C3\%ADpio\%20 de\%20Paranagu\%C3\%A1\%202016.pdf

Pindamonhangaba. (2015, 2 de outubro). Lei complementar no 51 de 2 de outubro de 2015. Institui o Plano de Mobilidade Urbana, define princípios, políticas e estratégias e instrumentos para o desenvolvimento municipal e dá outras providências. Recuperado em novembro de 2016, de http://www. pindamonhangaba.sp.gov.br

Poppeliers, R. J. M., \& Ricci, A. (2013). Review of the action plan on urban mobility. Zoetermeer: Panteia. Final Report. Research to Progress. 120 p.

Porto Alegre. Prefeitura Municipal. (2016). Plano diretor de mobilidade urbana. Empresa Pública de Transporte e circulação. Anexo 1. Recuperado em novembro de 2016, de http://lproweb.procempa.com.br/

Racero, J., Hernández, M., Guerrero, F., \& Racero, G. (2012). Design methodology and an integrated decisions support system for sustainable transportation plans. Sustainable Transport, 5 , 219-242.http://dx.doi.org/10.1007/978-3-642-23550-4_10.

Rio das Ostras. Prefeitura Municipal. (2015). Mobilidade urbana: plano de diretrizes. Recuperado em novembro de 2016, de http://www.riodasostras.rj.gov.br/noticia3264.html

Roojen, T. V., \& Nesterova, N. (2013). Applied framework for evaluation in CIVITAS PLUS II. CIVITAS WIKI. Coordination, evaluation and dissemination of CIVITAS PLUS II. Deliverable. 4.10. Recuperado em maio de 2015, de http://www.civitas. eu/sites/default/files/Results\%20and\%20Publications/ civitas_wiki_d4_10_evaluation_framework.pdf

Rudolph, F., Black, C., \& Glensor, K. (2014). How urban transport projects are appraised: current practice in the EU. Project Evidence. Economic benefits of sustainable transport. Wuppertal: Wuppertal Institute for Climate, Environment and Energy. Final version. Deliverable 2.2. 32 p. 
Santos. Prefeitura Municipal. (2015). Plano de mobilidade urbana. Recuperado em novembro de 2016, de http:// www.santos.sp.gov.br

São José do Rio Preto. (2015, 17 de abril). Lei no 11.736, de 17 de abril de 2015. Institui o Plano de Mobilidade Urbana do Município de São José do Rio Preto-SP. Recuperado em novembro de 2016, de www.riopreto.sp.gov.br/PortalGOV/ SubportaisDownload?tp=geral \&i=102838

São José dos Campos. Prefeitura Municipal. (2015). Plano Diretor de Mobilidade Urbana de São Josédos Campos. PlanMob 2015. Caderno Preliminar. Recuperado em novembro de 2016, de http://planmob.sjc.sp.gov.br/

São Paulo. Prefeitura Municipal. (2015). PlanMob SP/2015. Plano de Mobilidade de São Paulo. Recuperado em novembro de 2016, de http://www.prefeitura.sp.gov.br/cidade/secretarias/ upload/chamadas/planmobsp_v072_1455546429.pdf

Suárez, C. B., \& Llaneza, J. L. O. (2013). Evaluación del diseño de políticas públicas: propuestas de un modelo integral. Revista del CLAD Reforma y Democracia, (57), 37-66.

Tormans, H., Miermans, W., Cools, M., Polders, E., Janssens, D., \& Wets, G. (2013). Performance assessment of local mobility policy-making administrations using the principles of total quality management in Flanders, Belgium: expounding the decision-making processes. International Journal of Sustainable Transportation, 7(4), 318-346. http://dx.doi. org/10.1080/15568318.2011.633155.

Transporti e Territorio - TRT. (2010). Sustainable urban transport plans. Brussels: European Parliament, Policy Department.

Uberlândia. Prefeitura Municipal. Secretaria Municipal de Trânsito e Transportes. (2010). Plano Diretor de Transporte e Mobilidade de Uberlândia. Recuperado em novembro de 2016, de http://docplayer.com.br/6410497-Plano-diretorde-transporte-e-mobilidade-urbana-de-uberlandia.html

União da Vitória. (2015, 14 de julho). Lei no 4.526, de 14 de julho de 2015 (2015). Institui o Plano de Mobilidade Urbana de União da Vitória. Recuperado em novembro de 2016, de http://www.legislador.com.br/

United Nations - UN. (2014). World urbanization prospects: the 2014 revision: highlights. New York: United Nations.
Urbana Logística Ambiental do Brasil Ltda. (2015). Plano Municipal de Mobilidade Urbana. PlanMob 2015. Recuperado em novembro de 2016, de http://www.saoluizgonzaga. rs.gov.br/

Urbes Trânsito e Transportes. (2014). Plano Diretor de Transporte Urbano e Mobilidade. Plano de Mobilidade da cidade de Sorocaba. Recuperado em novembro de 2016, de https:// www.urbes.com.br/uploads2/PDTUM_CAMARA_SITE.pdf

Vargem Grande Paulista. Prefeitura Municipal. (2016). Plano de mobilidade urbana. Recuperado em novembro de 2016, de http://www.vargemgrandepta.sp.gov.br/

Venâncio Aires. (2014, 17 de abril). Lei Complementar n⿳o 077 de 17 de dezembro de 2014. Institui o Plano Diretor de Transportes e Mobilidade Urbana. PlanMob do Município de Venâncio Aires. Recuperado em novembro de 2016, de http://www.venancioaires.rs.gov.br

Via 11. Engenharia de Segurança Viária Ltda. (2016). Plano Municipal de Mobilidade Urbana de Concórdia (SC). Recuperado em novembro de 2016, de https://concordia.atende.net

Vieira, J., Moura, F., \& Viegas, J. M. (2007). Transport policy and environmental impacts: the importance of multiinstrumentality in policy integration. Transport Policy, 14(5), 421-432.http://dx.doi.org/10.1016/j.tranpol.2007.04.007.

Wefering, F., Rupprecht, S., Bührmann, S., \& Böhler-Baedeker, S. (2013) Guidelines: developing and implementing a sustainable urban mobility plan. Brussels: Rupprecht Consult, European Commission. $151 \mathrm{p}$.

Wolfram, M. (2004). Expert working group on sustainable urban transport plans. Germany: Rupprecht Consult. Final report. Deliverable D4. 106 p.

Wolfram, M., Bührmann, S., Martino, A., \& Brigati, E. (2005). Sustainable Urban Transport Plans (SUTP) and urban environment: policies, effects, and simulations: review of European references regarding noise, air quality and $\mathrm{CO}^{2}$ emissions. Germany: Rupprecht Consult. Final report. 240 p.

Recebido: Jul. 13, 2016

Aprovado: Jan. 17, 2017 